\title{
Genomics of lipid-laden human hepatocyte cultures enables drug target screening for the treatment of non-alcoholic fatty liver disease
}

Stephanie Breher-Esch ${ }^{1 \dagger}$, Nishika Sahini ${ }^{1 \dagger}$, Anna Trincone ${ }^{1}$, Christin Wallstab ${ }^{2}$ and Jürgen Borlak ${ }^{1 *}$ (D)

\begin{abstract}
Background: Non-alcoholic fatty liver disease (NAFLD) is a major health burden in need for new medication. To identify potential drug targets a genomic study was performed in lipid-laden primary human hepatocyte (PHH) and human hepatoma cell cultures.

Methods: PHH, HuH7 and HepG2 hepatoma cell cultures were treated with lipids and/or TNFa. Intracellular lipid load was quantified with the ORO assay. The Affymetrix HG-U133+ array system was employed to perform transcriptome analysis. The lipid droplet (LD) growth and fusion was determined by fluorescence microscopy. LD associated proteins were imaged by confocal immunofluorescence microscopy and confirmed by Western immunoblotting. Bioinformatics defined perturbed metabolic pathways.
\end{abstract}

Results: Whole genome expression profiling identified 227, 1031 and 571 significant regulated genes. Likewise, the combined lipid and TNFa treatment of PHH, HuH7 and HepG2 cell cultures revealed 154, 1238 and 278 differentially expressed genes. Although genomic responses differed among in-vitro systems, commonalities were ascertained by filtering the data for LD associated gene regulations. Among others the LD-growth and fusion associated cell death inducing DFFA like effector C (CIDEC), perilipins (PLIN2, PLIN3), the synaptosome-associated-protein 23 and the vesicle associated membrane protein 3 were strongly up-regulated. Likewise, the PPAR targets pyruvatedehydrogenase-kinase-4 and angiopoietin-like-4 were up-regulated as was hypoxia-inducible lipid dropletassociated (HILPDA), flotilin and FGF21. Their inhibition ameliorates triglyceride and cholesterol accumulation. TNFa treatment elicited strong induction of the chemokine CXCL8, the kinases MAP3K8, MAP4K4 and negative regulators of cytokine signaling, i.e. SOCS2\&SOCS3. Live cell imaging of DsRED calreticulin plasmid transfected HuH7 cells permitted an assessment of LD growth and fusion and confocal immunofluorescence microscopy evidenced induced LD-associated PLIN2, CIDEC, HIF1a, HILPDA, JAK1, PDK4 and ROCK2 expression. Notwithstanding, CPT1A protein was repressed to protect mitochondria from lipid overload. Pharmacological inhibition of the GTPasedynamin and the fatty acid transporter-2 reduced lipid uptake by 28.5 and 35\%, respectively. Finally, a comparisons of in-vitro/NAFLD patient biopsy findings confirmed common gene regulations thus demonstrating clinical relevance.

\footnotetext{
* Correspondence: Borlak.Juergen@mh-hannover.de

† Stephanie Breher-Esch and Nishika Sahini contributed equally to this work.

${ }^{1}$ Centre for Pharmacology and Toxicology, Hannover Medical School,

Carl-Neuberg-Str. 1, 30625 Hannover, Germany

Full list of author information is available at the end of the article
}

(c) The Author(s). 2018 Open Access This article is distributed under the terms of the Creative Commons Attribution 4.0 International License (http://creativecommons.org/licenses/by/4.0/), which permits unrestricted use, distribution, and reproduction in any medium, provided you give appropriate credit to the original author(s) and the source, provide a link to the Creative Commons license, and indicate if changes were made. The Creative Commons Public Domain Dedication waiver (http://creativecommons.org/publicdomain/zero/1.0/) applies to the data made available in this article, unless otherwise stated. 
(Continued from previous page)

Conclusion: The genomics of fat-laden hepatocytes revealed LD-associated gene regulations and perturbed metabolic pathways. Immunofluorescence microscopy confirmed expression of coded proteins to provide a rationale for therapeutic intervention strategies. Collectively, the in-vitro system permits testing of drug candidates.

Keywords: Non-alcoholic fatty liver disease (NAFLD), Transcriptomics, LD-associated proteins, Drug targets; nuclear receptor/PPARs, TNFa

\section{Background}

Non-alcoholic fatty liver disease (NAFLD) is a major health burden and characterized by intrahepatic lipid droplets of different size. An essential criterion for the histological definition of NAFLD is the presence of lipid droplets in $>5 \%$ of hepatocytes [1]. The prevalence of fatty liver disease ranges between 40 and 90\% in Western countries and is about $75 \%$ among obese individuals [2]. NAFLD is hallmarked by an imbalance in lipid homeostasis, abnormalities in mitochondrial metabolism, endoplasmic reticulum (ER) stress, impaired glucose tolerance, dyslipidemia and cardiovascular disease. Note, clinical evidence suggests NAFLD and the metabolic syndrome to be potentially linked with insulin resistance being a prerequisite for its development [3-5]. Therefore, a complex relationship exists between obesity, insulin resistance and NAFLD while the diagnostic criteria for the metabolic syndrome encompass a range of abnormalities, i.e. BMI > 30, impaired fasting glucose, impaired glucose tolerance, Type 2 diabetes, high blood pressure, elevated plasma triglycerides and HDL cholesterol as well as increased urinary albumin excretion [6]. Collectively, NAFLD and the metabolic syndrome involve a spectrum of conditions and when accompanied by inflammation results in liver fibrosis and cirrhosis that is a possible route to hepatocellular carcinoma (HCC) [7, 8]. Drug treatment may also induce hepatic steatosis or even exacerbates pre-existing NAFLD.

Given that over-nutrition can develop into obesity and obesity is a common cause of NAFLD, it is justified to briefly summarize lipid uptake. Under the influence of intestinal enzymes, e.g. lipase and amylase, dietary lipids and carbohydrates are digested to monoglycerides, fatty acids, glucose, fructose and monosaccharides. Prior to systemic circulation, the nutrients pass the intestinal barrier and in the case of lipid/cholesterol uptake chylomicrons are formed in enterocytes and are released via the lymphatic capillaries into the systemic circulation such as the portal and/or jugular vein. Chylomicrons are then modified by lipoprotein lipase to produce chylomicron remnants which are taken up by the liver and are further processed in hepatocytes for the synthesis of triglycerides (TG). Free fatty acids in the circulation are mostly bound to albumin. Notwithstanding, hepatocytes are also major sites for de novo lipid synthesis from glucose where pyruvic acid is formed through glycolysis and further processed to acetyl CoA, i.e. a key and versatile intermediate that enters a series of reactions to produce triacylglycerols [9]. Alternatively, acetyl CoA derived from glucose metabolism or mitochondrial fatty acid- $\beta$-oxidation is processed in the citric acid cycle for energy production, i.e. ATP synthesis. Mitochondrial glucose and fatty acid metabolism can be a major source for reactive oxygen species (ROS) that is detoxified via superoxide dismutase, catalase and glutathione dependent reactions. However, in hepatic steatosis enhanced lipid metabolism is associated with increased ROS production that might exceed the cellular capacity of ROS detoxification and as a result causes lipid peroxidation and mitochondrial dysfunction to lower considerably the energy output to peripheral tissues [10]. In the long term and through a vicious cycle that involves the peptide hormone adiponectin, fat-laden hepatocytes engage in lipogenesis rather than lipid metabolism, and lipid removal via VLDL secretion becomes insufficient to reduce effectively the hepatic intracellular lipid burden.

Recently, we summarized the molecular pathophysiology of lipid droplet formation in hepatocytes [4], and targeting the unfolded protein response offers new possibilities in the treatment of NAFLD. Specifically, hepatic steatosis induces ER stress and as a result the unfolded protein response [11]. This pathway involves complex signaling cascades aimed at restoring cellular homeostasis. However, prolonged hepatic steatosis disrupts the ER and secretory pathway homeostasis and is predominantly associated with insulin resistance, inflammation and apoptosis [12, 13]. Moreover, ectopic LD formation perturbs physical and functional links between mitochondria and ER to influence hepatocellular metabolism [14]. Several models have been proposed $[15,16]$ that support the biogenesis of lipid droplets from the ER. It will be worthwhile to investigate the role of oxidative and ER stress for a better understanding of the pathogenesis of fatty liver disease.

There is unmet need for new medications to treat fatty liver disease. Therefore, our study aimed at identifying perturbed metabolic pathways and LD-associated proteins as potential drug targets in fat-laden human hepatocyte cultures. We explored two different routes in the budding and growth of lipids droplets, i.e. fatty acid 
treatment of cell cultures and ER stress induced by dithiothreitol. We focused on lipid droplet associated gene regulations as putative drug target and equally considered adaptive responses to hepatic steatosis. Note, depending on the cell type the lipid droplet can be dressed with hundreds of proteins [17] and several of these proteins are interesting drug targets for the treatment of NAFLD. This includes Plin2, i.e. a key regulator of the unfolded protein response and ER stress [18]. Besides, the fusion of smaller lipid droplets (LD) to larger ones causes macrovesicular steatosis and eventually ballooned hepatocytes to worsen the condition of NAFLD. Thus, LD-fusion and its association with the endoplasmic reticulum were examined by live cell imaging and immunofluorescence microscopy in DsRED calreticulin plasmid transfected human hepatoma cells. As primary human hepatocytes are not routinely available and may involve donor specific responses, the genomics of lipid-laden human hepatoma cells $\mathrm{HuH7}$ and HepG2 were also compared to findings obtained with primary human hepatocyte cultures. Moreover, non-alcoholic steatohepatitis (NASH) is characterized by inflammation. Typically serum levels of the cytokine TNFa are increased in NASH patients and are shown to correlate with histologic scores of liver injury [19]. Therefore, we investigated the genomic responses of steatotic hepatocyte cultures to TNF $\alpha$ treatment.

Collectively, transcriptomics of fat-laden hepatocytes informed on NAFLD response genes and enabled the search for mechanistically linked and lipid droplet associated gene regulations. The study revealed putative and established drug targets with some targets being validated by confocal immunofluorescence microscopy and Western immunoblotting. Furthermore, clinical relevance of in-vitro NAFLD response genes was established by confirming their regulation in patient liver biopsies. Altogether, the employed research strategy permits the search for novel LD-associated targets for the pharmacological treatment of NAFLD and to test drug candidates to broaden the perspective of new medications.

\section{Methods}

Liver specimens from $N=6$ patients undergoing elective surgery for colorectal liver metastasis were used to isolate hepatocytes for their culture as detailed below. The basic patient characteristics are given in Additional file 1: Table S1. All tissue donors gave informed consent for experimental use of clinical data and liver specimen prior to surgery. Ethical approval for the protocol and use of liver samples was obtained from the Ethics Committee of the Hannover Medical School (Tr/L, 2499 and $\operatorname{Tr} / \mathrm{L}, 466$ 31309). Additionally, the human hepatoma cell lines HepG2 and HuH7 are established cell lines and are available through the American type culture collection (ATCC) and the Japanese Collection of Research Bioresources Cell Bank (JCRB0403) of the National Institutes of Biomedical Innovation, Health and Nutrition, Japan.

\section{Primary human hepatocyte cell culture}

Healthy specimens of liver section material were immediately transferred in ice cold physiologic saline solution to the laboratory, and hepatocytes were isolated as described previously [20,21]. Vessels visible on the cut surface were cannulated and the perfusion was initiated with $200 \mathrm{~mL}$ of an ethylene glycol tetraacetic acid (EGTA) -containing HEPES buffer at pH 7.4 and $37^{\circ} \mathrm{C}$. Subsequently, the perfusion was continued with $200 \mathrm{~mL}$ HEPES buffer. Thereafter, extracellular matrix degradation was achieved by perfusion with $200 \mathrm{~mL}$ of a HEPES buffer containing collagenase (Liberase $\mathrm{CI}$, Roche, Germany) and calcium chloride dihydrate at $37^{\circ} \mathrm{C}$. The enzyme containing perforate solution was recirculated. Following perfusion, the liver capsule was carefully removed, and cells were liberated by gentle shaking of the liver specimen in ice cold buffer containing Hanks buffered salt HEPES and bovine serum albumin. The resulting cell suspension was filtered through a nylon mesh and washed three times in buffer at $4{ }^{\circ} \mathrm{C}$. Viability of the hepatocytes was assessed by trypan blue exclusion and ranged between 78 and $94 \%$.

Primary human hepatocytes (PHH) were cultured in 6-well plates (Techno Plastic Products AG, Switzerland) in a collagen sandwich as described previously [21, 22] for up to 7 days. Approximately 1, 5 million hepatocytes were seeded per well and after attachment the non-adherent cells were removed and a second layer of collagen was applied on top of the cells. The PHHs were cultured with supplemented William's E culture medium (Lonza $\mathrm{GmbH}$, Germany) at $37^{\circ} \mathrm{C}$ with $100 \%$ humidity and $5 \% \mathrm{CO} 2$. The cell culture medium was changed daily and morphologically inspected by phase contrast microscopy.

\section{HepG2 and HuH7 cell culture}

The human hepatoma cell lines HuH7 and HepG2 were cultured in DMEM supplemented with $10 \%$ fetal calf serum, $1 \%$ glutamine and $1 \%$ pen- strep at $37{ }^{\circ} \mathrm{C}$ with $100 \%$ humidity and 5\% CO2. The cell culture media and reagents were purchased from Gibco, USA.

\section{Cell treatment}

At around $80 \%$ confluence cells were treated with 0.5 $\mathrm{mM}$ of an equimolar mixture of palmitic and oleic acid (PA/OA) and/or TNFa $(5 \mathrm{ng} / \mathrm{ml})$ for either $72 \mathrm{~h}$ or 7 days. The final DMSO concentration in control and PA/ OA treated cell cultures was $0.5 \% v / v$, and culture medium was changed daily. 


\section{ER stress induced LD formation}

$\mathrm{HuH} 7$ cells were seeded at $1 \times 10^{5}$ in a 24 well plate and transfected with ds RED calreticulin for $24 \mathrm{~h}$ using PolyFect transfection reagent from Qiagen according to the manufacturer's recommendations. Note, the ds RED calreticulin plasmid is the kind gift of Professor Naim, Institute of Biochemistry, University of Veterinary Medicine Hannover. Post transfection, cells were treated for $24 \mathrm{~h}$ with $10 \mu \mathrm{M}$ DTT (dithiotriol) and this reagent protects sulfhydryl groups of proteins against oxidation. The lipids were visualized with the fluorescent dye monodansyl pentane (MDP) for $15 \mathrm{~min}$, and live cell images were captured using the TiE Nikon microscope. The images were analyzed with the NIS elements software version 4.13

As mentioned above, the $\mathrm{HuH7}$ cells were transfected with the DsRED calreticulin plasmid and treated with 1:1 equal mixture of $0.5 \mathrm{mM}$ oleic (OA) and palmitic (PA) acid for $24 \mathrm{~h}$. Post treatment, cells were fixed with 4\% formalin and stained with MDP for $15 \mathrm{~min}$ and the coverslips were mounted using Mowiol. The images were captured using the Nikon Ti-E microscope.

\section{Imaging of intracellular lipid load}

Hepatocyte cultures were treated with 1:1 mixture of $0.5 \mathrm{mM}$ PA/OA for $1 \mathrm{~h}, 2 \mathrm{~h}, 4 \mathrm{~h}, 6 \mathrm{~h}, 24 \mathrm{~h}, 48 \mathrm{~h}$ and $72 \mathrm{~h}$. The cells were fixed with a $4 \%$ formalin solution, $60 \%$ isopropanol and stained with oil red $\mathrm{O}(\mathrm{ORO})$ according to the manufacturer's recommendation (Sigma Aldrich, Germany). The images were captured at a magnification of $20 \mathrm{x}$ by microscopy using appropriate filters for the ORO and DAPI nuclei stain.

\section{Intracellular lipid quantification}

Calibrates consisting of $0,10,25,50$ and $100 \mu \mathrm{g} / \mathrm{ml}(\mathrm{PA} /$ PO) were prepared and placed into disposable cuvettes. ORO was added and the absorbance was measured at $520 \mathrm{~nm}$ in a spectrophotometer according to the manufacturer's recommendation. A standard curve was fitted by plotting calibrates against absorbance readings. Typically, a correlation coefficient of $\mathrm{R}^{2}>0.975$ was obtained. To determine intracellular lipid content the media of lipid-laden hepatocyte was removed and the ORO staining of cell cultures was performed as described above. Excess dye was removed by repeated washing. Subsequently, intracellular ORO stained lipids are extracted by adding 4\% Igepal CA-630 in $1 \mathrm{ml}$ of isopropanol. The solution was measured in a spectrophotometer at $520 \mathrm{~nm}$ to quantify the concentration of ORO against the standard curve.

\section{Inhibition of FA transporters dynamin and FATP2}

HepG2 cell cultures $(1.2 \times 105$ cells/well $)$ were incubated at $37^{\circ} \mathrm{C}$ with DMEM (K-), DMSO $(\mathrm{K}+)$, PA/OA, PA/
$\mathrm{OA}+80 \mu \mathrm{M}$ dynamin blocker (Dynasore, Abcam, UK) and/or PA/OA and $9 \mu \mathrm{M}$ FATP2 blocker (CB16.2). The steatotic cell cultures were treated with dynasore and/or CB16.2 for $1 \mathrm{~h}$; the FATP2 blocker was kindly provided by Prof. Concetta C. DiRusso, University of Nebraska [23]. For the dynamin assay, culture medium without serum was used as serum inhibits the effect of the Dynasore while studies with CB16.2 to block FATP2 were conducted in the presence of serum.

Post treatment, cells were harvested, washed three times with PBS and fixed with $4 \%$ formaldehyde. After incubation at room temperature for $20 \mathrm{~min}$, the cells were washed with deionized water and treated with $60 \%$ isopropanol for 3-5 min and finally suspended in oil red $\mathrm{O}$ (ORO) stain (Sigma Aldrich, Germany) and incubated at room temperature for $10 \mathrm{~min}$. After staining, the cells were washed several times with deionized water to remove excess stain and re-suspended in $100 \mu \mathrm{l}$ running tap water. The ORO stain was imaged with Texas Red filter (excitation 562/emission624) by phase contrast fluorescence and light microscopy using the Nikon TiE microscope. Furthermore, quantification of the intracellular lipid was done as described before, and statistical testing involved a t-test and was considered significant at $p<0.05$.

\section{Immunofluorescence phase contrast live cell imaging}

The human hepatoma cells $\mathrm{HuH7}$ were incubated for $24 \mathrm{~h}$ with $1: 1$ mixtures of $0.5 \mathrm{mM}$ oleic and palmitic acid. Additionally, HepG2 cells were treated with a $0.5 \mathrm{mM}$ mixture of PA/OA for $3 \mathrm{~h}$ and LD fusion was assayed with propranolol at $200 \mu \mathrm{M}$. The images were captured by phase contrast fluorescence microscopy at 40x using time-lapse z-stack (Nikon TiE) and further analyzed using the NIS elements software version 4.13. Lipid droplets were visualized with the fluorescent MDP dye.

Immunofluorescence microscopy of PLIN2, CIDEC, CPT1A, HILPDA, HIF1a, JAK1, PDK4 and ROCK2

Perilipin 2 (PLIN2) was purchased from Antibodies Online (Aachen, Germany) (cat\# ABIN112185), the cell death-inducing DFFA-like effector c (CIDEC), CPT1A, HIF1 $\alpha$, JAK1 and Rock 2 were obtained from Santa Cruz Biotechnology, Inc. (cat\# sc-99,342, sc-139,480, sc-10,790, sc-277 and sc-5561). HILPDA was purchased from ProSci, Poway, USA (cat. 6491). Briefly, PHHs and $\mathrm{HuH7}$ cell cultures were treated with $0.5 \mathrm{mM}$ of an equal mixture of PA/OA for $24 \mathrm{~h}$, fixed with $4 \%$ formalin and permeabilised with $0.2 \%$ TritonX100 for $5 \mathrm{~min}$. The hepatocytes were incubated at room temperature in a dark chamber with primary antibody (1:200) for one hour and with the secondary antibody (1:50) for $45 \mathrm{~min}$. The lipid droplets were stained with the fluorescent dye monodansylpentane at $0.1 \mathrm{mM}$ for $15 \mathrm{~min}$ followed by mounting of the slides with Mowiol 4-88. Immunofluorescence 
microscopy was performed with the Nikon TiE phase contrast microscope as detailed above.

\section{Western blotting (WB) of PLIN2}

WB experiments were done with the Trans-Blot Turbo system according to the manufacturer's recommendations (Bio-Rad, Munich, Germany). After SDS-PAGE the gel was sandwiched within the blotting paper and the PVDF membrane and placed inside the cassette of the Trans-Blot Turbo Transfer System (Bio-Rad, Germany). Both the membrane and the blotting paper sheets were provided as ready-to-use in the Midi Format kit (Bio-Rad, Germany). Transfer of proteins was done with a protocol preset by the manufacturer, i.e. up to $25 \mathrm{~V}$ at constant amperage of $2.5 \mathrm{~A}$ for $7 \mathrm{~min}$. After blotting the gels were discarded and the successful transfer of proteins was confirmed by staining the membrane with Ponceau S solution $(0.1 \%$ in $5 \%$ acetic acid, Sigma Aldrich, Germany). The blotting membrane was placed in a 5\% milk powder solution dissolved in $1 \mathrm{X}$ Tris Buffered Saline $+0.1 \%$ Tween 20 (TBS-T) (TBS contains $50 \mathrm{mM}$ Tris- $\mathrm{HCl}$ ph 7.6 and $150 \mathrm{mM} \mathrm{NaCl})$. Membranes were gently shaken for $1 \mathrm{~h}$, and this procedure was followed by 3 washings with freshly made TBS-T lasting $10 \mathrm{~min}$ each in agitation. Primary antibodies were diluted in a proper amount of TBS-T and incubation of the antibody with the blotting membrane was done overnight at $4{ }^{\circ} \mathrm{C}$ in agitation. Thereafter, membranes were washed 3 times with $2.5 \%$ milk in TBS-T (10 min each) and the incubation of the secondary antibody was carried out for $1 \mathrm{~h}$ at room temperature after diluting the antibody in 5\% milk dissolved in TBS-T. Finally, 3 additional washings with TBS-T were done to remove unbound antibody.

Proteins were detected with the Clarity Western ECL Substrate kit (Bio-Rad, Germany). The kit contains two reagents that were mixed in a 1:1 ratio. The horseradish peroxidase conjugated to the secondary antibody catalyzes the reaction between luminol and hydrogen peroxide (contained in the solutions of the kits), providing chemiluminescence that can be detected by a CCD camera. Membranes were removed from the box and dried from the excess of TBS-T before adding homogeneously $800 \mu \mathrm{l}$ of the reagents mixture. The reagent was applied for $3 \mathrm{~min}$ in the dark before placing the membrane inside the Chemidoc MP system (Bio-Rad, Germany) to permit detection of the band and image acquisition. Exposure time was usually set automatically by the device.

\section{Statistical analysis of cell culture experiments}

Statistical significance testing was done with the GraphPad Prism Software (version 6.05) for Windows, GraphPad Software, La Jolla California USA, https://www.graphpad.$\mathrm{com} /$ ). Except for the $1 \mathrm{~h}$ time period where a Wilcoxon matched-pairs signed rank test was used to study lipid uptake all other cell culture experiments involved a Mann Whitney test. " denotes a significant $p$-value $<0.05,{ }^{* * * *} p<0.001$ and ${ }^{* * * * *} p<0.0001$.

\section{Microarray data analysis}

Total RNA was isolated with the Qiagen RNA purification kit according to the manufacturer's instructions (Qiagen, Hilden, Germany). cRNA was prepared following the Affymetrix Gene Chip Expression Analysis Technical Manual (Santa Clara, USA) [24] and whole genome transcript expression analysis was carried out with the Affymetrix Gene chip HG-U133 version 2.0 array [25]. Whole genome microarray data were normalized by applying the robust multi-array average (RMA) method using the Gene Expression Console software for background-adjusted and log-transformed perfectly matched individual probes. Genes annotated with unknown functions (i.e. "EST/hypothetical proteins" or "ORF of unknown functions") were removed from the dataset. Subsequently, the data were transferred to the Transcriptome Analysis Console (TAC) and t-test was performed for statistical analysis of differentially expressed genes by comparing data from vehicle controls versus lipid treated cell cultures. Additionally, the TAC software performs the Benjamini-Hochberg procedure to determine the false discovery rate (FDR) that was controlled at an alpha level of 0.05 . The FDR was considered when determining statistically significantly regulated genes at $p<0.05$. The data were filtered for 300 genes which are considered to be mechanistically relevant in the process of lipid droplet biogenesis in hepatocytes as recently published by us [4]. The microarray data is available through the GEO Omnibus public repository (accession number: GSE122660).

\section{Results \\ Lipid droplet formation induced by ER stress}

The DsRED calreticulin plasmid was used to visualize ER. After successful transfection of $\mathrm{HuH7}$ cells the cultures were treated with $10 \mu \mathrm{M}$ DTT for $24 \mathrm{~h}$. Note, dithiothreitol blocks disulfide-bond formation and causes ER stress to result in ectopic LD formation (Fig. 1a\&b). The inserts depicted in Fig. 1 are images of single LDs stained with the fluorophore monodansylpentane (in blue); the region in pink indicated by arrows illustrates an apparent association with the ER as hallmarked by the DsRED fluorophore. Furthermore, the calreticulin plasmid was studied in HepG2 cell but the transfection efficiency was low (data not shown). Given that $\mathrm{HuH7}$ cells are larger as compared to HepG2 cells the lipid droplet/ER association could be better visualized in the HuH7 cell line. We also investigated ectopic LD formation in cultures of primary human hepatocytes 

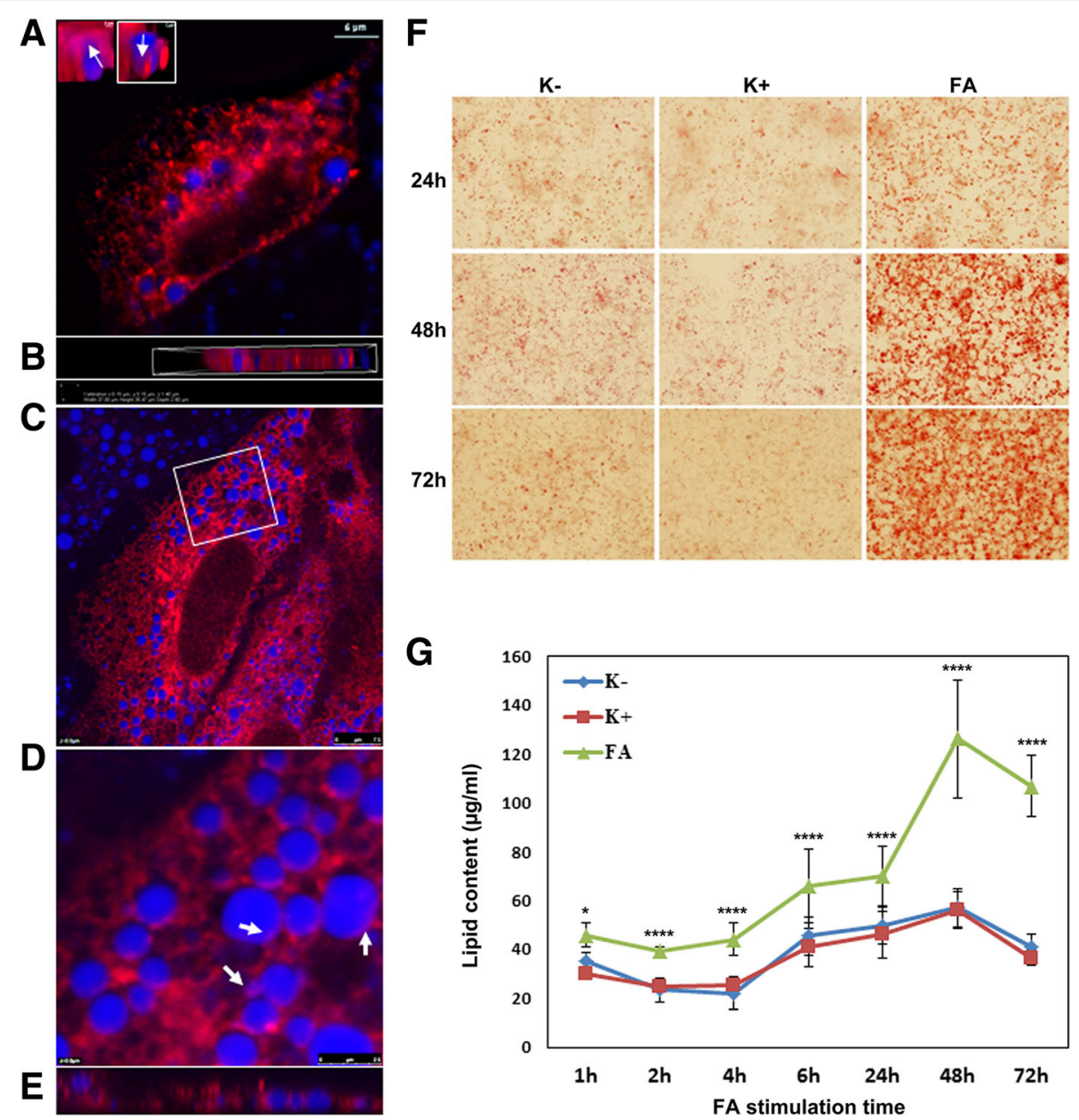

Fig. 1 ER stress induced LD formation and quantification of intracellular hepatic lipid content in HepG2 cells. a \& b Depicted are HuH7 cells transfected with ds RED calreticulin. The cultures were treated with $10 \mu \mathrm{M}$ DTT for $24 \mathrm{~h}$. The lipid droplets were stained with the fluorophore MDP (blue) and live cell images were captured with the TiE Nikon microscope. The images were analyzed with the NIS elements software version 4.13 and images in the inset are single LDs. Regions in pink (as indicated by arrows) appear to be associate with ER. The scale bar is $6 \mu \mathrm{m}$. Panel B is a view of panel A in $x-y-z$ plane. c-e Fatty acid induced ER stress. Depicted are live cell images of HuH7 cells transfected with ds RED calreticulin. The cultures were treated with 1:1 equal mixture of oleic acid (OA) and palmitic acid (PA) for $24 \mathrm{~h}$. The images were captured using a Leica SP5 confocal microscope. The scale bar is $7.5 \mu \mathrm{m}$. Panel $\mathrm{D}$ is a zoomed image of the rectangular marked region shown in panel $\mathrm{C}$ and panel $\mathrm{E}$ depicts the Y-plane of the white dotted line shown in panel D. The arrows indicate the region where LDs appear to be associated with ER. $\mathbf{f}$ Phase contrast images of intracellular lipid droplets. Shown is the time dependent growth of LDs after FA stimulation. HepG2 cells were either treated with the (K+) or without (K-) DMSO vehicle control or PA/OA (FA) for 24, 48 and $72 \mathrm{~h}$. LDs are visualized with the ORO stain. Images were captured with a Nikon TiE phase contrast fluorescent microscope using the NIS elements software version 4.13. $\mathbf{g}$ Time dependent increase in intracellular lipid content of HepG2 cells treated with 1:1 mixture of $0.5 \mathrm{mM} \mathrm{PA} / \mathrm{OA}$ for up to $72 \mathrm{~h}$. Further information is given in Additional file 2: Figure S1, Additional file 3: Figure S2, Additional file 4: Figure S3. * denotes a significant $p$-value $<0.05$ and ${ }^{* * * *} p<0.0001$

but the DTT treatment proved to be toxic and did not permit image analysis.

\section{Lipid droplet formation induced by fatty acid treatment} Similar results were obtained after lipid treatment of HuH7 cells. Here, LDs appeared to be associated with ER (regions in pink indicated by arrows in Fig. 1c-e). The kinetics of intracellular lipid uptake in hepatoma and primary human hepatocyte cultures was investigated; a significant difference between the DMSO vehicle and palmitate-oleate-treated cell cultures was observed. Using the oil red $\mathrm{O}(\mathrm{ORO})$ stain the lipids were quantified at $1 \mathrm{~h}, 2 \mathrm{~h}, 4 \mathrm{~h}, 6 \mathrm{~h}, 24 \mathrm{~h}, 48 \mathrm{~h}$ and $72 \mathrm{~h}$ following fatty acid treatment, and the total intracellular lipid concentration increased from $45(1 \mathrm{~h})$ to $70 \mu \mathrm{g} / \mathrm{ml}$ after $24 \mathrm{~h}$. Figure if depicts phase contrast images of total intracellular hepatic lipid content visualized with the ORO stain; the kinetics of lipid uptake is depicted in Fig. 1g. When compared to the DMSO vehicle control the intracellular lipid content increased further by about 80 and $70 \mu \mathrm{g} / \mathrm{ml}$ at 48 and $72 \mathrm{~h}$, respectively. Similar results were obtained for the $\mathrm{HuH} 7$ 
and primary human hepatocyte cultures (data not shown). See Additional file 2: Figure S1 for additional information.

\section{Live cell imaging of LD growth}

As described previously [26, 27] LD fusion events involve transfer of lipids and other LD components from one LD to the other and may grow by expansion or coalescence. To determine LD growth $\mathrm{HuH7}$ cells were treated with a $1: 1$ mixture of $0.5 \mathrm{mM}$ oleic acid and palmitic acid for $24 \mathrm{~h}$ and studied by live cell imaging. Heterotypic LD fusion was observed where smaller LDs (orange arrows) fuse with larger ones (red arrows) (Additional file 3: Figure S2A-E).

Alike, steatotic HepG2 cells were treated for $3 \mathrm{~h}$ with propranolol to trigger LD fusion. Note previous studies have shown that the $\beta$-adrenoceptor antagonist propranolol functions as a fusogen possibly through interaction with the phospholipid monolayer [28]. As depicted in Additional file 4: Figure S3 the live cell images show a clear demarcation between the untreated and propranolol treated HepG2 cells. The fatty acid treated cells were filled with LDs and when treated with propranolol the cells show larger but fewer LDs. As a result multiple lipid droplets randomly grew in size, and the LD fusion events were captured showing mostly heterotypic fusion events with differing radii of LDs as was observed in the fatty acid treated $\mathrm{HuH} 7$ cells (Additional file 4: Figure S3B). The entire event was recorded for $15 \mathrm{~min}$. Post $3 \mathrm{~h}$ of propranolol treatment the cells were apoptotic and hence could not be used to evaluate gene expression changes.

Importantly, recent research evidenced propranolol to worsen liver injury in a mouse model of NASH via activation of death pathways. Therefore, the drug should be given to NAFLD patients with caution [29].

\section{Inhibition of hepatic lipid uptake}

Recently the DiRussco lab reported inhibition of fatty acid transport by CB16.2 (=Lipofermata) and CB5 (=Grassofermata) with an IC50 of $6.7 \pm 0.6$ and $6.3 \pm$ $09 \mu \mathrm{M}$, respectively [30-32].

As a proof-of-concept pharmacological inhibition of the fatty acid transport protein 2 (FATP2) by CB16.2 and of dynamin, which functions as a large GTPase in vesicle transport and the regulation of endocytosis, was investigated in human hepatoma cell cultures. For this purpose HepG2 cells were treated with either media control (K-), the DMSO vehicle control $(\mathrm{K}+)$, with PA/ OA (lipid) or with lipid and inhibitors of fatty acid uptake for $1 \mathrm{~h}$. Subsequently, the intracellular lipids were stained with ORO (Fig. 2 A1 \& B1), and the total lipid content was quantified in a spectrophotometer at 520 nm (Fig. 2 A2 \& B2) as described in the Material and
Method section. The images (Fig. 2 A1 \& B1) were captured at $10 \mathrm{x}$ magnification by phase contrast microscopy and analyzed using the NIS elements software version 4.13.

When compared to PA/OA treated cells the intracellular lipid content was significantly reduced by 28.5 and $35 \%$ using dynasore or CB16.2, respectively. Note, both are small molecule inhibitors of dynamin and FATP2 (Fig. 2 A2 \& B2). Previous studies demonstrated that treatment of Hela cells and macrophages with $80 \mu \mathrm{M}$ dynasore blocked dynamin activity effectively and reduced total cholesterol uptake by about 15\% [33]. In the present study lipid uptake in HepG2 cell cultures was also significantly inhibited by dynasore (Fig. 2 A2). It is well known that dynasore interferes with the regulation of endocytosis by blocking dynamin, i.e. a crucial molecule for clathrin-dependent coated vesicle formation [34, 35]. While long chain fatty acids (LCFA) are taken up via caveolae mediated endocytosis [36] blocking the process of endocytosis could effectively reduce influx of fatty acids into hepatocytes. Similarly, inhibition of FATP2 activity by CB16.2 resulted in 35\% reduction in FA uptake of HepG2 cells (Fig. 2 B2).

\section{Whole genome transcriptome profiling}

We investigated genomic responses to steatosis and inflammation in cultures of primary human hepatocyte and hepatoma cell lines. Altogether 227, 1031 and 571 genes were significantly regulated in $\mathrm{PHH}, \mathrm{HuH} 7$ and HepG2 cell cultures, respectively after treatment with fatty acids for $72 \mathrm{~h}$. Equally, the combined treatment with lipid and TNFa caused significant regulation of 154, 1238 and 278 DEGs in PHH, HuH7 and HepG2 cell cultures. Bioinformatics revealed perturbed metabolic pathways but also adaptive responses to hepatic steatosis. Subsequently, the data were filtered for DEGs considered to be mechanistically linked to lipid droplet biogenesis (Additional file 5: Table S2) [4]. Shown in Fig. 2c\&d are Venn diagrams to inform on LD associated gene regulations. Specifically, hydroxysteroid dehydrogenase like 2 (HSDL2) is commonly up-regulated among the different cell culture systems after $72 \mathrm{~h}$ of lipid treatment. The protein functions in the transport and metabolism of fatty acids and cholesterol esters. Its dual localization in mitochondria and peroxisomes was confirmed by confocal microscopy [37]. Several genes were commonly up-regulated when at least two cell culture systems were compared (Fig. 2c) and included the carnitine palmitoyltransferase $1 \mathrm{~A}(\mathrm{CPT} 1 \mathrm{~A})$, a rate-limiting enzyme in the $B$-oxidation of long chain fatty acids. However, expression of the protein was reduced to protect mitochondria from lipid overload as evidenced by immunofluorescence microscopy (see below). Likewise, the delta-9 stearoyl-coA desaturase (SCD) was up-regulated. Inhibition of the ER localized enzyme 


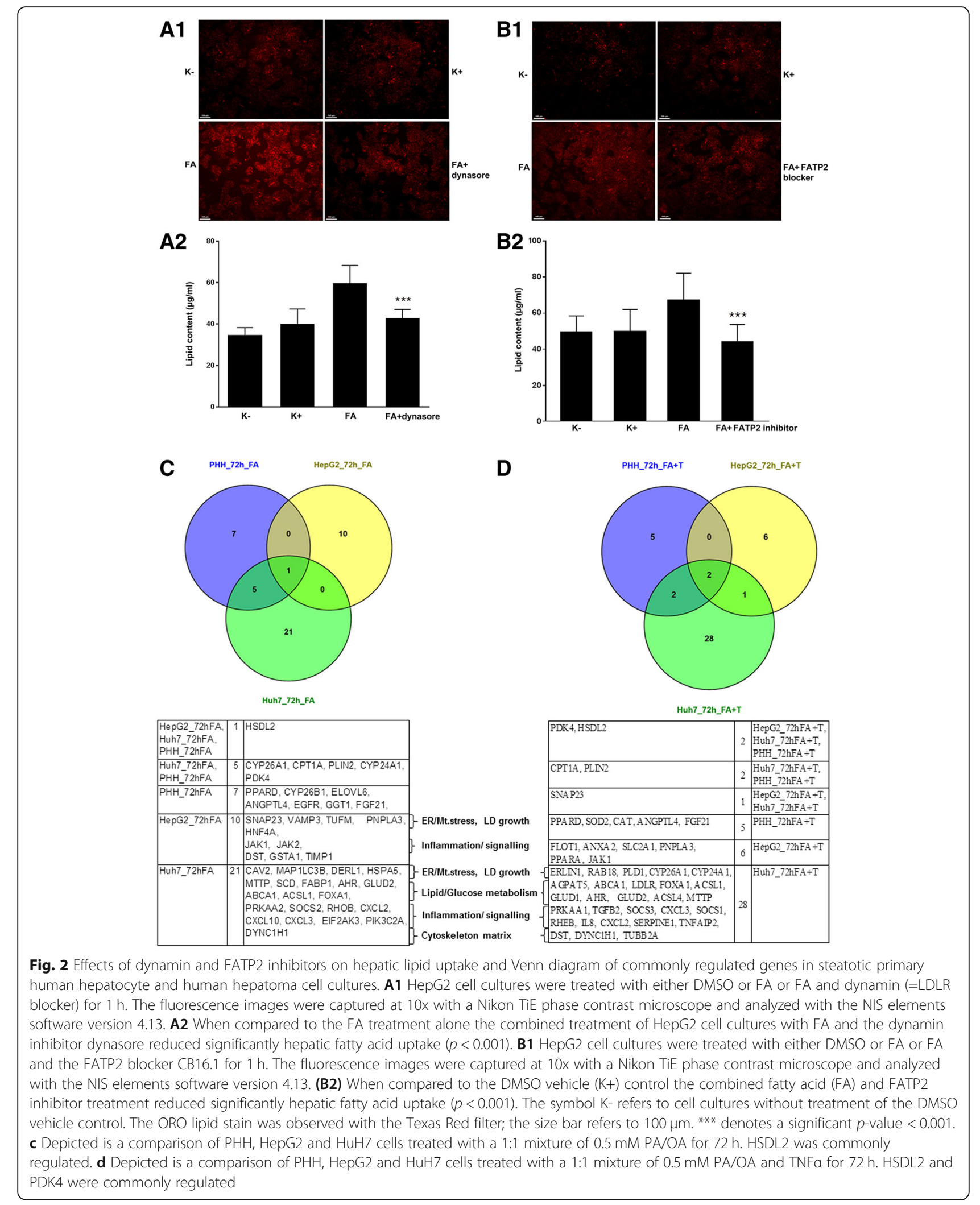


increases insulin sensitivity and resistance to diet-induced obesity [38]. Further examples include the peroxisome proliferator-activated receptor $\operatorname{PPAR} \beta / \delta$ and its targets ANGPTL4 as well as pyruvate dehydrogenase kinase, isozyme 4 (PDK4) whose expression were significantly up-regulated in cultures of primary human hepatocytes after lipid treatment for $72 \mathrm{~h}$. The induction of perilipin 2 and PDK4 exemplifies the suitability of the in-vitro system to investigate drug candidates for the treatment of hepatic steatosis. Specifically, expression of perilipin 2 and PDK4 is augmented in LD biogenesis with PDK4 inhibiting the pyruvate dehydrogenase complex. Hif1 $\alpha$ promotes PDK4 gene expression and although not significantly changed at the transcript level Hif $1 \alpha$ protein expression was strongly induced particularly after the combined lipid and TNF $\alpha$ treatment (see below immunofluorescence microscopy). Therefore, mechanistic aspects in the mitochondrial pathogenesis of hepatic steatosis can be investigated. Likewise induction of the monooxygenases CYP24A1, CYP26A1 and CYP26B1 informs on retinoic and fatty acid oxidations. The regulation of the ras homolog family member B (RHOB) alerts to changes in cytokinesis and microtubule-dependent signaling and induced expression of the microsomal triglyceride transfer protein (MTTP) signifies increased cholesterol biosynthesis and triglyceride accumulation. Conversely, induction of FOXA1 highlights adaptive responses to hepatic steatosis. This transcription factor was shown to reduce lipid accumulation in human hepatocytes and to repress lipid uptake by inhibiting the fatty acid transporter FATP2 [39]. A further example of an adaptive response is the significant up-regulation of fibroblast growth factor 21. There is clear evidence for FGF21 signaling to ameliorate lipotoxicity, inflammation and to improve mitochondrial function [40].

\section{Combined lipid and TNFa treatment}

To mimic inflammation cell cultures were treated with lipid and TNF $\alpha$. Importantly, increased serum TNF $\alpha$ levels correlate with histologic scores of liver injury in NASH patients [19]. A comparison of the genomic responses among different cell culture systems revealed HSDL2 and PDK4 to be commonly regulated (Fig. 2d). Additionally, genes commonly up-regulated in at least two culture systems included CPT1 $\alpha$ as well as the lipid droplet associated PLIN2 and SNAP23, the latter facilitating lipid droplet fusion. The transcriptional up-regulation of CPT1 $\alpha$ and PPAR $\alpha$ HepG2, $72 \mathrm{~h}$ ) indicate coordinate responses to influence fatty acid uptake and $\beta$-oxidation in mitochondria. Conversely, the up-regulation of MTTP and ANGPTL4 imply increased cholesterol ester biosynthesis and triglyceride accumulation in hepatocytes. The low density lipoprotein receptor (LDLR) was also up-regulated in Huh7 treated cell cultures and induced CYP24A1 and CYP26A1 monooxygenases are part of vitamin $\mathrm{D}$ and retinoic acid signaling. Note induced CYP24A1 expression may provide a rational for the common observation of vitamin $\mathrm{D}$ deficiency in NAFLD patients [41]. Additionally, annexin A2 was up-regulated (HepG2, $72 \mathrm{~h}$ ) and the coded protein functions in cell motility, cytoskeleton and endocytosis.

To further evaluate genomic responses genes $\geq 3$-fold regulated genes were considered (Fig. 3, panel A and B). With primary human hepatocyte cultures the genes coding for PDK4, PLIN2, ANGPTL4 and FGF21 were commonly up-regulated after lipid and the combined lipid and TNFo treatment (Fig. $3 \mathrm{~A} 1$ and B1). The significant induction of PDK4 alters mitochondrial glucose and fatty acid metabolism; its activity is influenced by PPAR $\beta / \delta$ and HIF1 $\alpha$ [42]. ANGPTL4 is a direct PPAR $\beta / \delta$ target and was shown to participate in glucocorticoid-regulated triglyceride metabolism by inhibiting lipoprotein lipase-mediated plasma triglyceride clearance [43, 44]. Previous research also demonstrated ANGPTL4 to increase cholesterol synthesis in the liver [45].

The up-regulation of fibroblast growth factor 21 is of great importance. FGF21 treatment of mice was shown to correct obesity [46] and to improve insulin sensitivity and glucose control in rodents and humans [47]. Therefore we consider FGF21 regulation as an adaptive response to hepatic steatosis. Improving FGF21 signaling in NAFLD patients enables novel treatment options. Conversely, repression of the hepatic glucose transporter GLUT2 implies an imbalance in glucose uptake and osmoregulation (Fig. 3 A1).

The combined lipid \& TNF $\alpha$ treatment elicited strong induction of CXCL8, MAP3K8, MAP4K4, SOCS2 and SOCS3. Note, SOCS2 deletion was shown to protect against hepatic steatosis but worsens insulin resistance in high-fat-diet-fed mice [48] while ablation of SOCS3 enhances hepatic insulin sensitivity but increases lipogenesis resulting in fatty liver and obesity [49]. Furthermore, up-regulation of CXCL8 is of critical importance for the recruitment of neutrophils and was shown to cause tissue damage in NAFLD/NASH [50]. Inhibiting CXCL8 or blocking its receptor CXCR1 may proof to be effective in the treatment of NASH [51]. The up-regulation of MAP3K8 and MAP4K4 highlights responsiveness of the in-vitro system to cytokines. Pharmacological inhibition of MAP3K8 was reported to block human cytotoxic $\mathrm{T}$ lymphocyte effector functions [52] and may be therapeutically explored in the treatment of NASH. Likewise MAP4K4 inhibitors are explored in different disease indications linked to inflammation and cancer. Inducible deletion of Map4k4 in obese mice improved insulin sensitivity in liver and adipose tissue [53]. Further examples include repressed dystonin (DST) coding for a cytoskeletal protein and 


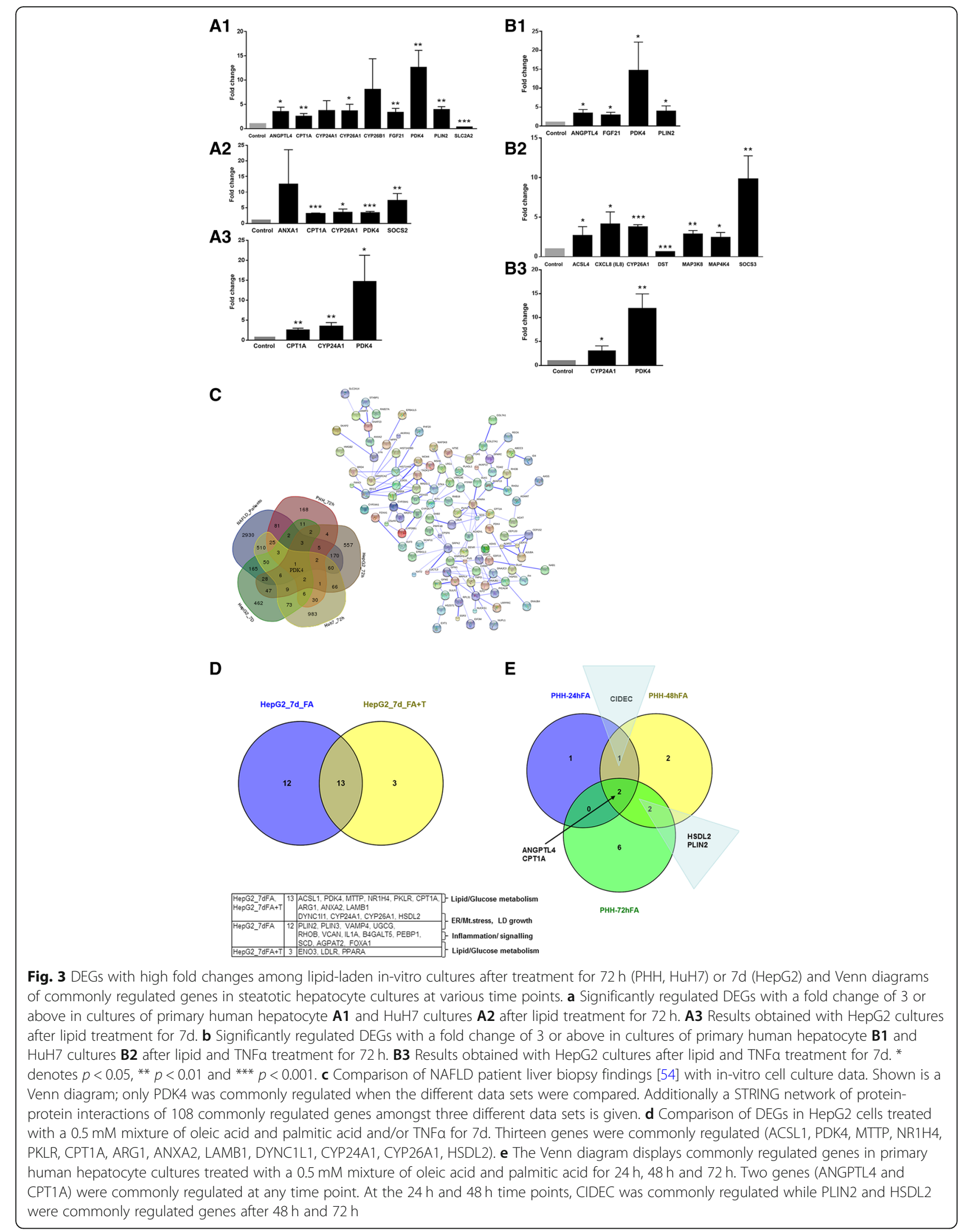


induced ACSL4, an enzyme that regulates fatty acid synthesis and lipid degradation (Fig. 3 B2).

We considered transcript changes of HepG2 cultures after daily lipid treatment for 7 days and observed sustained induction of CPT1 $\alpha$, CYP24A1 and PDK4 (Fig. 3 A3 and B3). The combined lipid and TNF $\alpha$ treatment elicited 13 common gene regulations. Transcriptional regulation of ACSL1, PDK4, MTTP, NR1H4 and PKLR highlight altered lipid and glucose metabolism while DYNC1L1, CYP24A1, CYP26A1 and HSDL2 point to ER/mitochondrial stress and LD growth (Fig. 3d). We also considered the time dependent changes in LD associated gene regulations in cultures of primary human hepatocytes (Fig. 3e). Two genes (ANGPTL4 and CPT1 $\alpha$ ) were expressed at any given time point while the gene coding for the cell death-inducing DFFA-like effector C was significantly induced at $24 \mathrm{~h}$ and $48 \mathrm{~h}$; expression of PLIN2 and HSDL2 was prominent at $48 \mathrm{~h}$ and $72 \mathrm{~h}$ (Fig. 3e). Specifically, CIDEC is an LD associated protein to support LD growth by transferring neutral lipids from smaller to larger LDs and may interact with the perilipin 2 as observed by confocal immunofluorescence microscopy in co-localization studies (see below).

\section{Lipid-laden hepatocytes/NAFLD patient liver biopsy comparison}

We recently reported the genomics of fatty liver disease in human NAFLD [54], and the data were compared to findings obtained from lipid-laden hepatocytes. Figure 3c depicts a Venn diagram and reveals the kinase PDK4 to be commonly regulated among all experimental conditions once again highlighting the importance of PDK4 in inhibiting the pyruvate dehydrogenase complex in hepatic steatosis. Additionally, DEGs regulated alike among the different experimental data sets were selected for protein-protein interaction network analysis. Here, a total of 108 statistically significantly regulated genes were considered (Additional file 6: Table S3) and are shown to participate in 155 protein-protein interactions. Therefore, evidence was obtained for mechanistically linked and lipid droplet associated genes to interact with each other when the coded proteins were considered (String network analysis; Fig. 3c).

\section{Immunofluorescence microscopy}

We selected the targets CIDEC, CPT1A, HILPDA, HIF1 $\alpha$, JAK1, PDK4, PLIN2 and ROCK2 to further evaluate expression of the protein and therefore druggability by immunofluorescence imaging. Additionally, multicolor imaging studies were performed to probe for co-localization of CIDEC and PLIN2 on lipid droplets (Figs. 4, 5). Shown in Fig. 4 panel A is the immunofluorescence imaging of CIDEC (green channel) and PLIN2 (red channel). The lipid droplets are stained with the blue fluorescent dye monodansylpentane. Note the marked expression of CIDEC and PLIN2 in lipid-laden primary human hepatocyte cultures. Shown in panel B is the co-localization of CIDEC and PLIN2 on lipid droplets in proximity to each other. A similar picture emerged after the combined lipid and TNFo treatment of PHHs (Fig. 4, panel C) and both proteins dress the LD monolayer in proximity. Conversely, CPT1A is markedly reduced in steatotic hepatocyte cultures, and a similar reduction in CPT1A protein was observed after the combined lipid and TNFa treatment of PHHs (Fig. 4, panel D). It is tempting to speculate that the reduction in CPT1A protects mitochondria against lipid overload. Notwithstanding, CPT1A mRNA was induced in expression (Fig. 3, panel A1-A3) in PHHs and hepatoma cell lines after lipid but not the combined lipid and TNF $\alpha$ treatment.

We next considered the expression of HILPDA and Rock2 (Fig. 5). Although insignificantly changed at the mRNA level both targets are of critical importance in LD biogenesis (HILPDA) and actin cytoskeleton dynamics (ROCK2). Note, the combined lipid and TNFa treatment of HuH7 cells significantly repressed dystonin mRNA (see Fig. 3b) and this protein functions as an intermediate filament of the cytoskeleton. Immunofluorescence microscopy revealed marked expression of HILPDA (panel A) and the kinase ROCK2 (panel B) in lipid treated Huh7 cell cultures; however the combined lipid and TNF $\alpha$ treatment diminished their expression.

We also investigated regulation of HIF1 $\alpha$, JAK1 and PDK4 by immunofluorescence microscopy in cultures of primary human hepatocytes (Fig. 6, panels a-c) and observed induced expression of the proteins in lipid-laden hepatocytes particularly by the combined lipid and TNF $\alpha$ treatment.

Additionally, we performed WB experiments (Fig. 7, panel A) in hepatocyte cultures and found PLIN2 to be strongly induced by about 77 and 124-fold after lipid and the combined lipid \& TNF $\alpha$ treatment. Note the distinct association of PLIN2 with the monolayer of the lipid droplets shown in Fig. 7 panel B. Lastly, a summary of major findings is given in Fig. 7c.

\section{Discussion}

Fatty liver disease has become a major health burden in Western societies and there is unmet need for new medication. To facilitate the drug discovery process and to search for new drug targets a gene expression profiling study was performed. Based on our recent review on the molecular pathophysiology of lipid droplet formation in hepatocytes the genomic data were filtered for approximately 300 genes mechanistically linked to lipid transport, lipogenesis, LD growth, glucose and fatty acid metabolism as well as signaling events [4]. Besides, lipid 

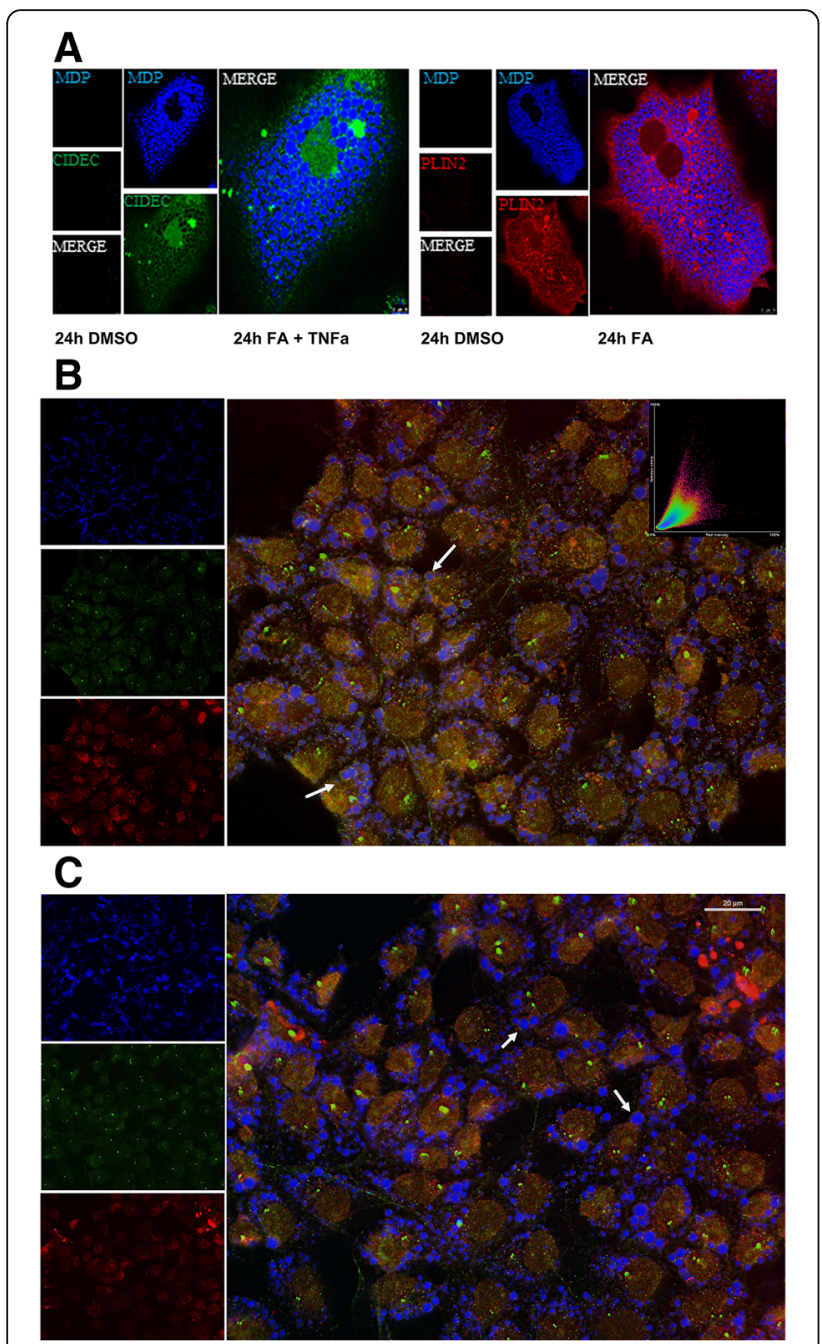

D

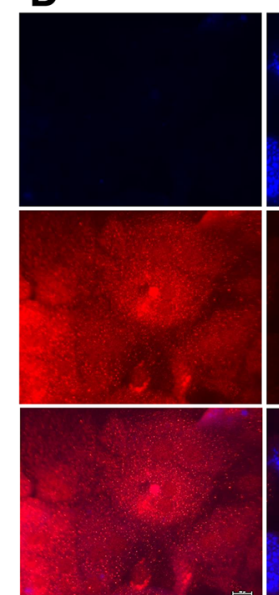

24 DMSO

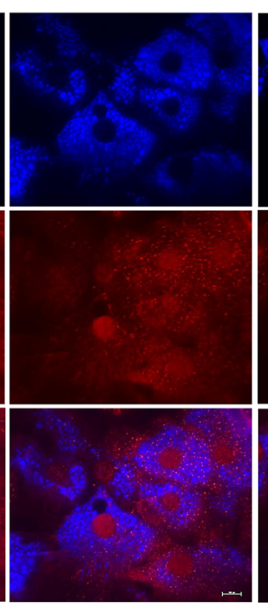

24h FA

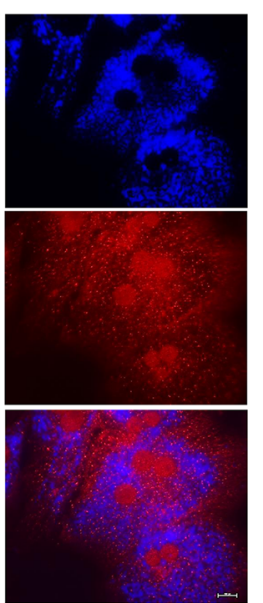

24h FA+TNF
Fig. 4 Immunofluorescence imaging of PLIN2, CIDEC and CPT1A protein expression in steatotic primary human hepatocyte cultures. a Primary human hepatocyte cultures were treated with a mixture of $0.5 \mathrm{mM}$ PA/OA and stained individually for PLIN2 or CIDEC. Lipids were stained with the blue fluorescent dye MDP. CIDEC is given in green; PLIN2 expression is shown in red. b Co-localization of PLIN2 and CIDEC on the surface of LD monolayers. Primary human hepatocyte cultures were treated with a mixture of $0.5 \mathrm{mM}$ PA/OA for $24 \mathrm{~h}$ and lipids were visualized the blue fluorescent dye MDP. Immunofluorescent staining of PLIN2 and CIDEC is given in the red and green channel, respectively. The co-localization image analysis was computed with a Pearson's correlation coefficient of 0,806,395, a Manders overlap $=0,942,398$ with K1; K2 $=0,751,118 ; 1,182,389$ and $a$ co-localization coefficient = 1:1. A scatter blot of the co-localization fluorescent signals is given as an insert. c Co-localization of PLIN2 and CIDEC on the surface of LD monolayers. Primary human hepatocyte cultures were treated with a mixture of $0.5 \mathrm{mM}$ PA/OA and TNFa for $24 \mathrm{~h}$ and lipids were visualized the blue fluorescent dye MDP. Immunofluorescent staining of PLIN2 and CIDEC is given in the red and green channel, respectively. The bar represents

$20 \mu \mathrm{m}$. d Immunofluorescence imaging of carnitine

palmitoyltransferase 1A (CPT). Primary human hepatocyte cultures were treated with a mixture of $0.5 \mathrm{mM} \mathrm{PA} / \mathrm{OA}$ for $24 \mathrm{~h}$. Lipids were stained with the blue fluorescent dye MDP and CPT1A is shown in red. The images were captured with the Nikon TiE phase contrast immunofluorescent microscope with a $60 x$ objective using the NIS elements software (version 4.13.); the scale bar is given in $\mu \mathrm{m}$. The merged channels are given as large images and single channels are shown as smaller images

growth over time was studied, and the morphological changes of lipid-laden hepatocyte cultures mimic, at least in part, changes observed in-vivo. We also evaluated inhibitors of FATP2 and dynamin in lowering hepatic intracellular lipid concentrations. Altogether, LD-associated gene regulations and perturbed metabolic pathways were identified, and LD associated proteins such as perilipin 2 were confirmed by immunofluorescence microscopy dressing the LD monolayer. Therefore, applying genomics to lipid-laden hepatocytes helped to pinpoint putative drug targets and to ascertain adaptive responses to hepatic steatosis. Due to the fact that primary human hepatocyte cell cultures are not routinely available the genomics of lipid-laden human hepatoma cells was also compared to findings obtained with $\mathrm{PHH}$. Furthermore, we investigated the effects of TNF $\alpha$ treatment to mimic inflammation as observed in steatohepatitis. Altogether known and putative drug targets were identified including the LD-growth and LD-fusion associated PLIN2, PLIN3, SNAP23 VAMP and the kinases PDK4, JAK, MAPK and ROCK2. Moreover, clinical relevance was established by comparing the genomic data from in-vitro studies with findings obtained from NAFLD patient biopsies.

Induction of hepatic steatosis and lipid droplet growth Initially, two approaches were employed to induce steatosis either by treatment with DTT, a reducing agent to 


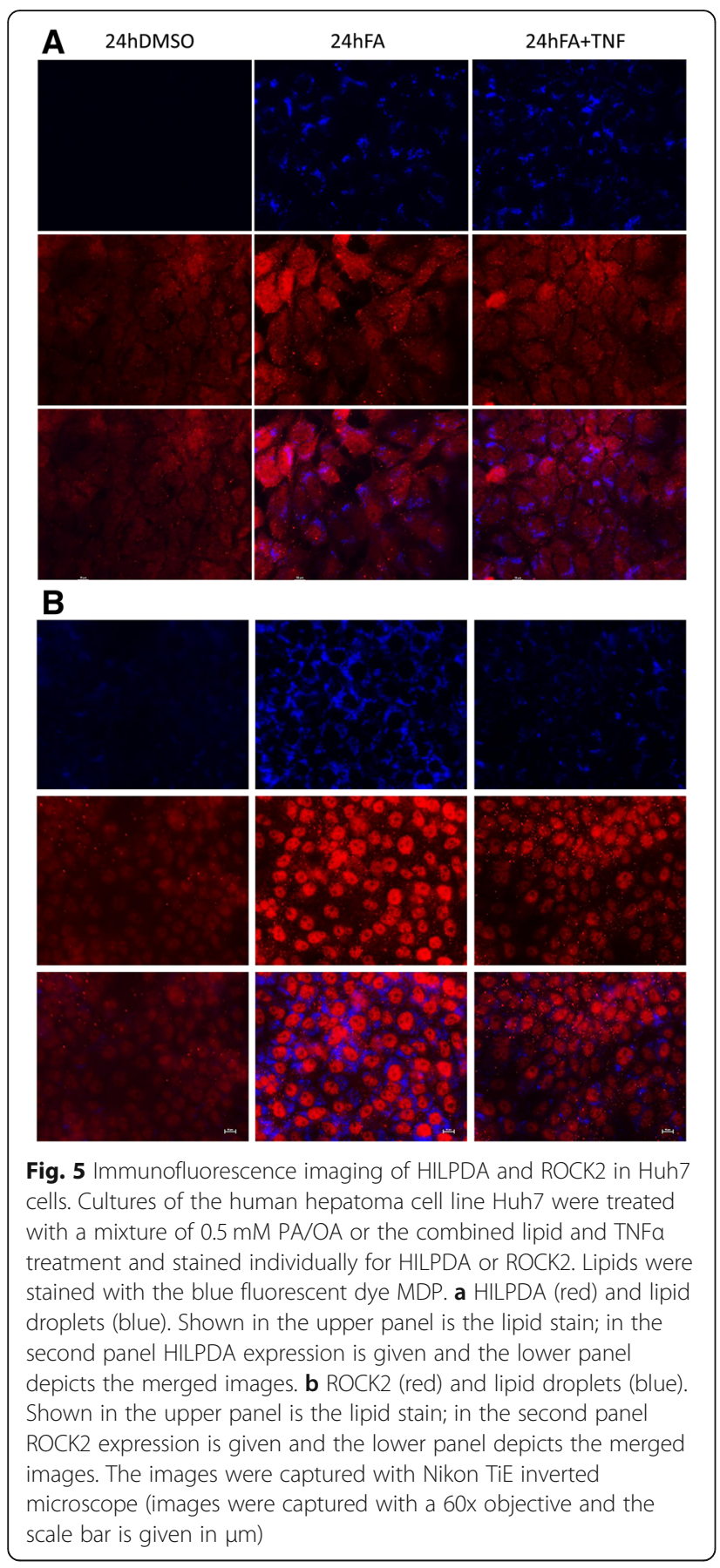

cause ER stress and ectopic LD budding or by treatment of hepatocyte cultures with a mixture of palmitic and oleic acid. Both treatments resulted in lipid droplet formation (Fig. 1). However, DTT even at lower concentrations was highly toxic and induced smaller sized LDs whereas PA/OA treatment was well tolerated and enabled an assessment of LD fusion by live cell imaging. Importantly, over-nutrition, lack of satiety and the resultant obesity can lead to fatty liver disease while consumption of a high-fat diet rapidly exacerbates the development of NAFLD especially at elevated glucocorticoid levels [55]. In order to mimic a high-fat "Western diet" the PA/OA treatment was considered in detail and the intracellular lipid content was quantified over time (Fig. 2). Specifically, one hour after fatty acid treatment the intracellular lipid content was significantly increased (Fig. 2 and Additional file 1: Figure S1). Apparently the hepatic lipid uptake followed two phases, i.e. the period $4 \mathrm{~h}$ to $24 \mathrm{~h}$ with a gradual increase and 24 $\mathrm{h}$ to $48 \mathrm{~h}$ where a steep increase but subsequent decline was observed at $72 \mathrm{~h}$. Supplementing the culture media with the LD fusogen propranolol accelerated LD growth and fusion (Additional file 3: Figure S3B) and this ß-adrenoceptor antagonist worsens liver injury in a model of non-alcoholic steatohepatitis [29].

The morphological features of steatosis and steatohepatitis include ballooning of hepatocytes; stressed organelles affect ER, mitochondria and peroxisome functioning [4, 56]. Additionally, the large number of intracellular LD influences cytoskeletal dynamics and signaling events. We observed on average 50 lipid droplets per cell after treatment with PA/OA for $24 \mathrm{~h}$. After $48 \mathrm{~h}$ and $72 \mathrm{~h}$ of treatment lipid droplets grew in size but declined in number. This was accompanied with an increase in hepatocyte surface area (data not shown). Collectively, the morphological changes of lipid-laden hepatocyte cultures mimic, at least in part, changes observed in-vivo. Moreover, the LD growth and fusion associated gene regulations (PLIN2, PLIN3, SNAP23, VAMP etc.) provide evidence for the relevance of the in-vitro model to recapitulate changes observed in-vivo.

\section{In-vitro hepatic steatosis response genes}

The genomic responses to lipid treatment were investigated in PHH and hepatoma cell lines. Note, hepatoma cell lines are easy to use and for a given cell line data reproducibility is robust. Conversely, primary human hepatocytes $(\mathrm{PHH})$ are difficult to obtain, costly and next to donor dependent responses $\mathrm{PHHs}$ can only be cultured for a short period of time. Consequently, there are important tradeoffs that need to be considered when comparing the different in-vitro systems. Although genomic responses to steatosis differed among the in-vitro systems commonalities were observed when the data were filtered for genes mechanistically linked to lipid droplet formation and metabolism (Additional file 5: Table S2). Hydroxysteroid dehydrogenase like 2 was commonly regulated among all systems, and the protein functions as desaturase in mitochondria and peroxisomes [37].

A comparison of in-vitro genomic data and human NAFLD biopsy findings (Fig. 3c) revealed the mitochondrial pyruvate dehydrogenase kinase 4 (PDK4) as commonly up-regulated. This kinase is of key importance in 

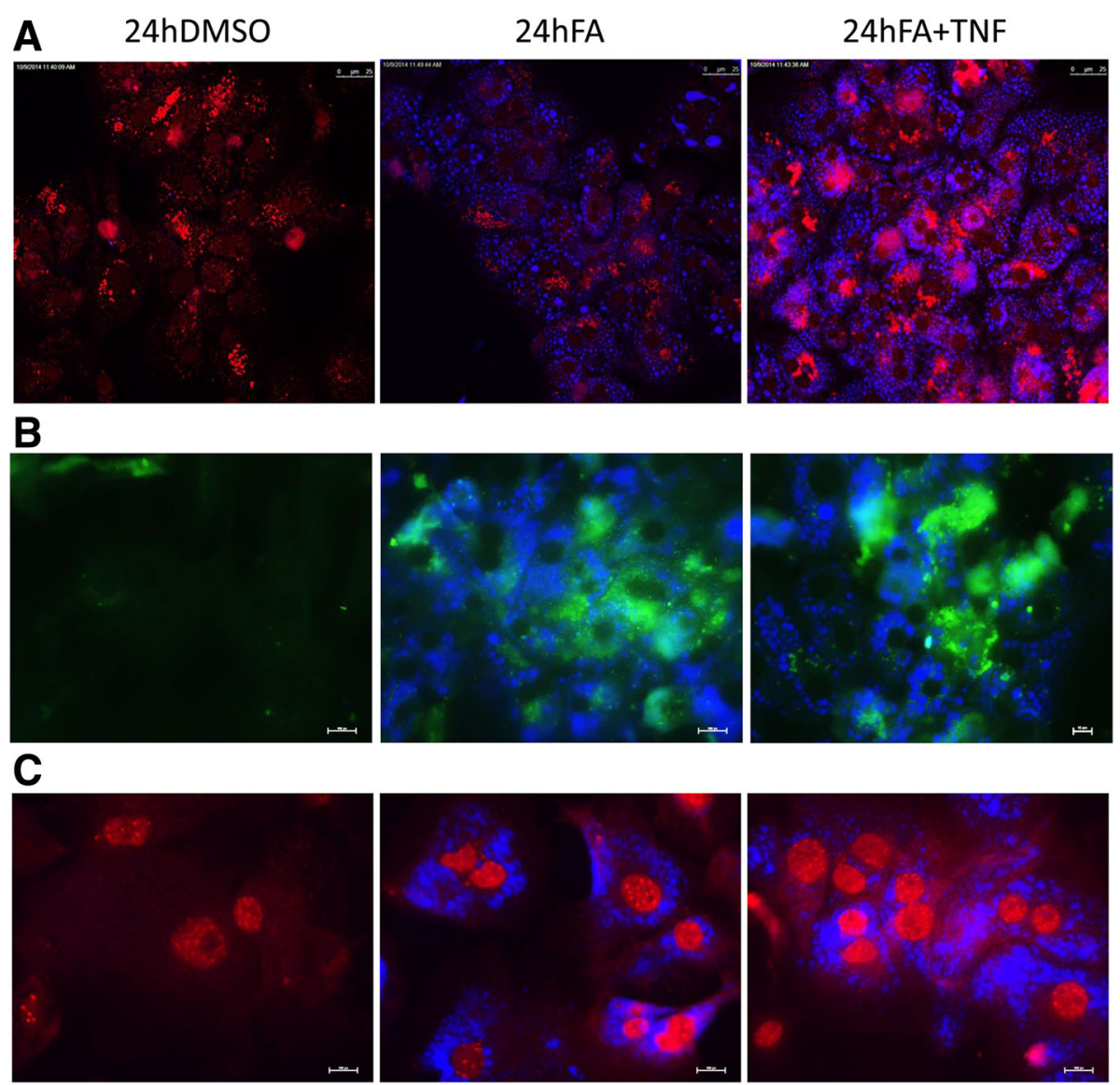

Fig. 6 Immunofluorescence imaging of Hif1, PDK4 and JAK1 in primary human hepatocyte cultures. Cultures of primary human hepatocytes were treated with a mixture of $0.5 \mathrm{mM}$ PA/OA or the combined lipid and TNFa treatment and stained individually for HIF1a, PDK4 or JAK1. Lipids were stained with the blue fluorescent dye MDP. Depicted are merged images. a HIF1a (red) and lipid droplet (blue). b PDK4 (green) and lipid droplets (blue). c JAK1 (red) and lipid droplets (blue). The images were captured with Nikon TiE inverted microscope (images were captured with a 60x objective and the scale bar is given in $\mu \mathrm{m}$ )

glucose metabolism. Expression of PDK4 is influenced by HIF1 $\alpha$ and retinoic acid and the retinoic acid hydroxylase CYP26A was significantly up-regulated in lipid-laden hepatocyte cultures. Previous research demonstrated CYP26A1 and CYP26B1 to be induced in expression by all-trans retinoic acid [57-59]. Furthermore, CYP monooxygenases catalyze oxidization of fatty acids. The induced expression of PPAR $\alpha$ in HepG2 cells and PPAR $\beta / \delta$ in primary human hepatocyte cultures stimulates PDK4 activity which in turn inhibits the conversion of pyruvate to acetyl-CoA. Next to PDK4 other target of PPAR $\beta / \delta$ such as ANGPTL4 and PLIN2 were significantly up-regulated. Induction of perilipin 2 is typically augmented in LD biogenesis as observed in cultures of primary human hepatocytes and the hepatoma cell lines (Fig. 4). Conversely, the glucose transporter 2 was significantly repressed in expression indicating an imbalance in glucose uptake and osmoregulation. The protein facilitates bidirectional glucose transport, and the complex interactions between glucose and lipid metabolism in fatty liver disease have been the subject of several reviews [4, 60]. Interestingly, Exendin-4, i.e. a glucagon-like peptide-1 receptor agonist improved fatty liver disease in ob/ob mice by regulating glucose transporter expression [61]; alike SREBP1c mediates glucose-stimulated GLUT2 gene expression in hepatocytes [62]. With cultures of primary human hepatocytes significant induction of angiopoietin-like 4 was observed that can be explained by the combined activity of PPAR $\beta /$ $\delta$ and HIF1 $\alpha$ [42]. Angiopoietin-like 4 was also shown to be a direct target of glucocorticoid receptor and participates in glucocorticoid-regulated triglyceride metabolism [43]. Induction of the growth factor FGF21 in PHH cultures is of great importance. This growth factor is predominantly secreted from the liver and ameliorates fatty liver disease through activation of the AMPK-SIRT1 pathway [63]. Another NAFLD regulated gene is carnitine palmitoyltransferase $1 \mathrm{~A}$. The coded protein is part of the carnitine shuttle in transferring long chain fatty acids to the inner mitochondrial membrane to initiate the process of fatty acid $ß$-oxidation. Unlike induced expression of 

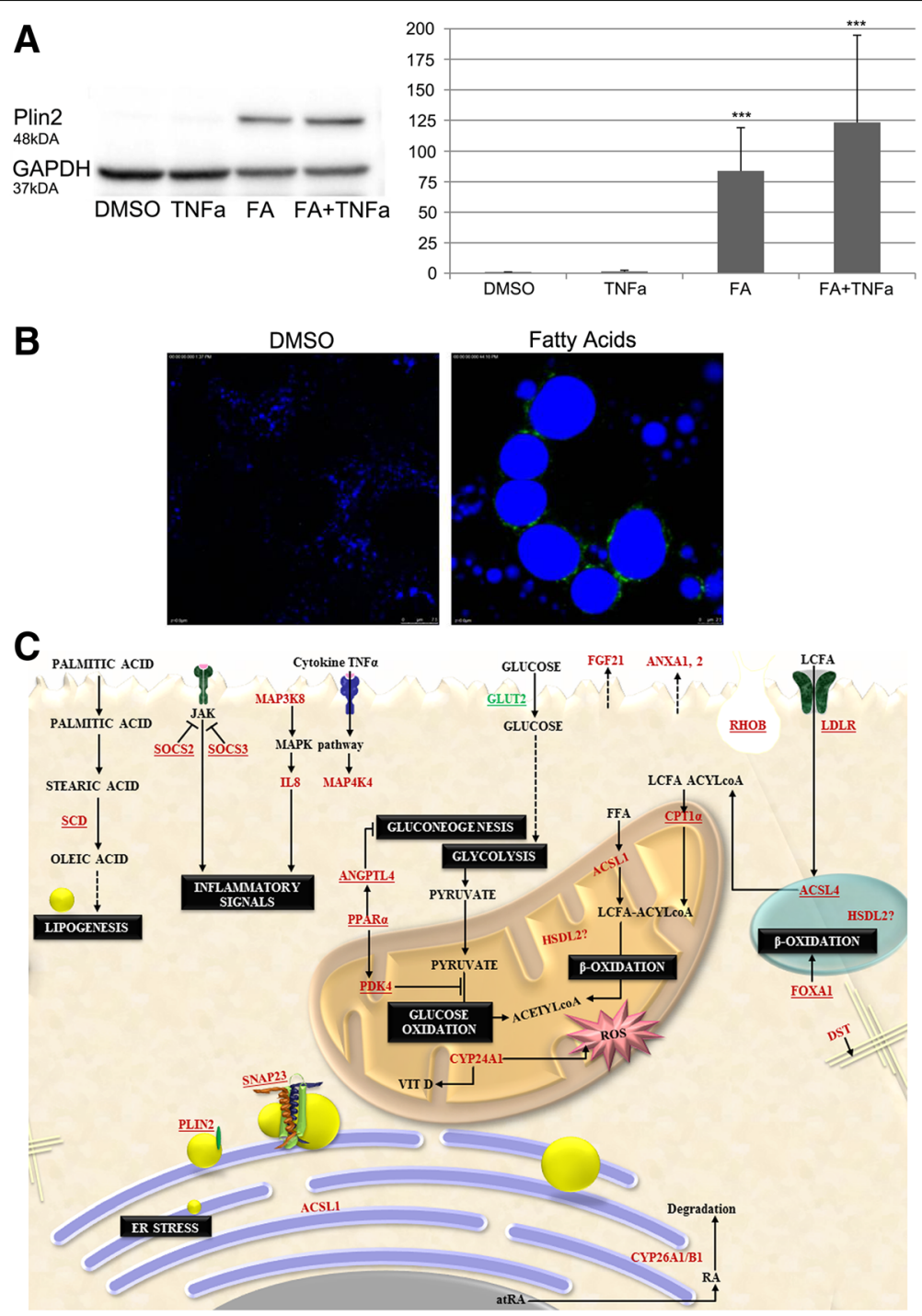

Fig. 7 Western blotting and immunofluorescence imaging of PLIN2 and a schematic overview of LD associated gene regulations in lipid-laden hepatocytes. Cultures of primary human hepatocyte and hepatoma cell lines were treated with a mixture of $0.5 \mathrm{mM}$ PA/OA or the combined lipid and TNFa treatment. a Western blotting of Plin2 with GAPDH as loading control.*** $p<0.01$. b Immunofluorescence of DMSO vehicle and lipid treated hepatocytes highlighting PLIN2 localization around the monolayer of LDs. The lipids were stained with MDP dye and the scale bar is $7.5 \mu \mathrm{m}$. c Overview of mechanistically linked and LD associated genes in an in-vitro model of fatty liver disease. Depicted is a summary of NAFLD responsive genes in steatotic hepatocyte cultures. Fatty acid uptake occurs either by diffusion or lipid transporters. Continuous supply of lipids leads to an imbalance in glycolysis and gluconeogenesis that in turn affects $\beta$-oxidation of fatty acids. The excess lipids are stored in the form of lipid droplets. Simultaneously, genes responsible for LD growth are up-regulated and support LD expansion. The hepatocytes also respond to external stimulus such as TNFa signals by up-regulating mitogen activated protein kinases as well as other inflammatory signals such as interleukins and suppressors of cytokine signaling. Overall, cellular homeostasis is disturbed leading to enhanced lipid synthesis and altered metabolic signaling and eventually augments lipotoxicity. Figure $7 \mathrm{c}$ had been created by the authors

CPT1A transcripts the protein was repressed (Fig. 4d) possible to protect mitochondria from lipid overload.

Figure 7 depicts a summary of the study findings highlighting the following results:

Steroyl coA desaturase is induced in lipid-laden hepatocyte cultures. The ER localized enzyme catalyzes the $\delta 9$ desaturation of fatty acyl-CoA substrates including palmitoyl- and stearoyl-CoA [38]. It is a key enzyme for the biosynthesis of monounsaturated fatty acids which are essential building blocks for the synthesis of hepatic TG and cholesterol esters and are exported via the VLDL secretory pathway. During LD growth PLIN2 expression is augmented and a recent study evidenced Plin2 to inhibit cellular glucose uptake through interactions with SNAP23, a SNARE complex protein [64]. Specifically, LD fusion is supported by the SNARE complex 
and SNAP23 is part of it. This vesical-membrane fusion protein was identified as frequently up-regulated in our genomics study of human NAFLD [54]; its common regulation signifies the importance of SNARE proteins in facilitating lipid droplet fusion with implication on glucose uptake and insulin sensitivity as suggested by Boström and colleagues [65].

During an initial phase mitochondrial $ß$-oxidation of fatty acids is increased in hepatic steatosis. Testimony to an altered mitochondrial lipid metabolism is the up-regulation of CPT $1 \alpha$ mRNA, i.e. a component of the carnitine acyltransferase system. However, the CPT1A protein was reduced in expression possibly to protect mitochondria from excessive lipid metabolism. Additionally, the peroxisomal acyl-CoA synthetase ACSL4 catalyzes activation of arachidonic and eicosapentaenoic acid [66]; its up-regulation influences synthesis of prostaglandins but also peroxisomal $\beta$-oxidation of long chain fatty acids to shorter ones while increased expression of ACSL1 and CPT1 $\alpha$ supports fatty acid metabolism in mitochondria. A recent report suggests overexpression of ACSL1 to correct mitochondrial dysfunction with increased coupling efficiency and decreased proton leakage [67]. Moreover, CPT1 $\alpha$ as well as ACSL1/ACSL4 may modulate activity of transcription factors by influencing intracellular availability of fatty acids and acyl-CoA derivatives which are ligands for various transcription factors including hepatic nuclear factor $4 \alpha$ and PPARs $[68,69]$. The present study revealed up-regulation of $\operatorname{PPAR} \beta / \delta$ and well known target genes of this transcription factor, i.e. PDK4, ANGPTL4 and PLIN2.

To mimic the condition of inflammation hepatocyte cell cultures were treated with lipids and TNF $\alpha$. Among the different systems the human hepatoma cell line $\mathrm{HuH7}$ was most responsive with significant up-regulation of the genes coding for SOCS3, CXCL8 and mitogen activated protein kinases. Note, liver-specific suppressor of cytokine signaling-3 deletion in mice enhanced hepatic insulin sensitivity but increased lipogenesis to result in fatty liver disease and obesity [49]. Alike, CXCL8 was significantly increased in NASH patients as compared to bland steatosis or healthy controls [70] thus demonstrating clinical relevance of the current findings with hepatocyte cultures. Furthermore, induction of FOXA1 is an adaptive response to hepatic steatosis and was shown to reduce lipid accumulation in human hepatocytes [39] as is the induction of FGF21 with its great therapeutic potential in the treatment of NAFLD [40].

\section{Pharmacological inhibition of lipid uptake}

Next to passive diffusion fatty acid uptake is dependent on transporters/translocases as well as receptor mediated mechanisms. Additionally, hepatic lipid uptake may involve lipid-rafts, calveolin- and clathrin mediated endocytosis. We therefore investigated the effects of FATP and dynamin inhibitors on hepatic lipid uptake by treating hepatocyte cultures with the small molecules CB16.2 and dynasore. Importantly, the fatty acid transporter FATP2 is expressed in the liver, and this protein is unique in a sense as it additionally functions as an acyl-CoA synthetase to support bile acid synthesis [71]. FATP2 augments uptake of long-chain fatty acids (LCFAs); therefore it regulates intracellular fatty acid pool size in hepatocytes [72]. In response to FATP2 inhibition lipid uptake in HepG2 cells was significantly reduced by $35 \%$ (see Fig. 2 b) and a similar $30 \%$ reduction in hepatic lipid uptake was observed with dynasore (Fig. 2a). Interestingly, dynasore was found in a screen of $\sim 16,000$ compounds aimed at identifying inhibitors of GST-Grb2-stimulated dynamin2 [34], and this GTPase is of critical importance in endocytosis and intracellular cholesterol transport [33, 73]. Our study confirms fatty acid uptake to involve fatty acid transport proteins and lipid mediated endocytosis. The in-vitro systems can therefore be used to investigate experimental inhibitors effectively.

\section{Genomic responses to hepatic steatosis reveal bona fide drug targets}

The genomic studies revealed a range of rational and putative drug targets. Specifically, we observed up-regulation of the microsomal triglyceride transfer protein (MTTP). In 2012 Lomitapide was approved as the first in class MTTP inhibitor for the treatment of family hypercholesterolemia. The genomic study also revealed regulation of the nuclear receptors PPAR $\alpha$ and $\mathrm{PPAR} \beta / \delta$ in addition to CPT 1 . Importantly, clofibrate and fenofibrate activate the PPAR $\alpha$ transcription factor and are well established lipid lowering agents with fenofibrate being used for decades in the clinic. Thus, the therapeutic benefit of this drug target was already demonstrated. Alike, the transcription factor PPAR $\beta / \delta$ is a key regulator of glucose and lipid metabolism, and different agonists have been developed to increase lipid metabolism and to improve insulin sensitivity as well as serum lipid profiles [74]. The present study revealed the PPAR $\beta / \delta$ targets ANGPTL4, PDK4 and HILPDA as highly interesting proteins for the development of inhibitors. Recently, a PDK2 inhibitor targeting the ATP binding pocket was reported to improve glucose tolerance and to reduce hepatic steatosis by affecting pyruvate dehydrogenase complex activity [75]. Research also identified HILPDA to inhibit adipose triglyceride lipase thus providing a rationale to inhibit activity of this protein in NAFLD [76]. Furthermore, topical inhibitors of PPAR $\beta /$ $\delta$ are developed to treat psoriasis [77]. Conversely, Etomoxir is an irreversible inhibitor of CPT1 and blocks fatty acid uptake and fatty acid ß-oxidation in 
mitochondria. This drug was evaluated in patients with heart failure and type 2 diabetes; however, clinical trials were terminated due to severe hepatotoxicity. Treatment of primary human hepatocyte cultures with lipids caused a marked reduction in CPT1A protein as evidenced by immunofluorescence (Fig. 4d). Note, a recent study demonstrated recovery of CPT1A to ameliorated hepatic steatosis in mice after treatment with the hepatic stimulator substance (HSS). A similar result was obtained when steatotic HepG2 cells were transfected with a plasmid for HSS and the effect was enhanced by C75, a CPT-1 activator [78]. Moreover, increased hepatic mitochondrial fatty acid oxidation reversed insulin resistance and glucose intolerance in obese mice independently of hepatic steatosis [79].

Additional file 5: Table S2 informs on NAFLD responsive genes which are regulated in common in in-vitro/ in-vivo comparisons. Several of the coded proteins are bona fide therapeutic targets and include the FOXO proteins. The FOXO1 transcription factor plays a decisive role in glucose and lipid metabolism. Inhibition of its activity by AS1842856 effectively lowers lipogenesis. The observed regulation of AKT and FOXO3 is also suggestive for mTORC1-independent regulation of autophagy as summarized by Ward et al. [80]. FOXO inhibitors are therefore a hot topic in development of new drugs for the treatment of metabolic disorders [81, 82].

The present study identified induced PDK4 in response to hepatic steatosis and this kinase functions as a metabolic switch between glucose and fatty acid metabolism. Independent studies revealed PDK4 activity to be influenced by thiazolidinediones and pioglitazone was shown to improve liver histology and fibrosis in patients with non-alcoholic steatohepatitis [83].

Lipid droplet associated proteins and their inhibition have become the focus of recent research [84] and the present study evidences exclusive expression of perilipin 2 and 3 in hepatic steatosis with PLIN2 being commonly regulated in-vitro and in-vivo (Additional file 5: Table S2). Importantly, rational drug design based on 3D crystallography revealed 4-Nitrophenyl 2,3,4-Tri-O-levulinoyl-Î $\pm-D$-mannopyranoside as an inhibitor for perilipin 1 [85] while perilipin 2 inhibition is considered to be a novel strategy in treating NAFLD and other age-related diseases [86].

Consistently, we observed regulation of flotilin-1; this lipid-raft associated protein is of critical importance in the budding of LDs and their growth. Given that endocytosis of flotillin-1 and flotillin-2 is regulated by Fyn kinase [87] its pharmacological inhibition may proof to be beneficial in treating progressive disease and particularly NASH. Moreover, among commonly regulated hepatic steatosis genes is FGF21. This growth factor belongs to a group of signaling proteins and was shown to ameliorate the condition of NAFLD. In a recent review the application of FGF21 analogues for the treatment of metabolic disorders and NAFLD has been summarized [88, 89].

Up-regulation of the LDL receptor in response to lipid treatment was another important finding and inhibition of LDLR internalization by dynasore was effective in lowering the intracellular lipid content as demonstrated in the present study and as reviewed by Preta et al. [90]. There are also reports demonstrating beneficial effects of L- and N-type calcium channel blocker on glucose and lipid metabolism in patients with hypertension and Type II diabetes [91].

These and other examples demonstrate the utility of the genomic approach in identifying novel drug targets for the treatment of fatty liver disease.

\section{Study limitations}

Although some key aspects of hepatic steatosis such as lipid droplet formation can be investigated in cultures of human hepatocytes the following caveats need to be considered: In-vivo progressive fat accumulation of the liver evolve through different mechanisms, i.e. over-nutrition, drug induced, ER stress etc. and eventually stimulates the release of pro-inflammatory chemokines either by stressed adipocytes or injured hepatocytes which cannot be precisely mimicked in a cell culture system. Additionally, TNF $\alpha$ treatment of lipid-laden hepatocytes mimics inflammation with TNF $\alpha$ serum levels being elevated in $\mathrm{NASH}$ patients. However, in-vivo the immune response augmented by Kupffer cells perpetuates acinar/lobular inflammation and involves mobilization and infiltration of neutrophils into the sinusoidal space to contribute to disease progression which cannot be studied in-vitro. Moreover, intrahepatic CD8 and natural killer T cells aggravate the condition of NASH and cytokine stress with alarm signals sent by harmed hepatocytes to stimulate Toll like receptor signaling and an immune response. The complex interactions between endothelial cells of the sinusoids and hepatic stellate cells, their activation to myofibroblasts within the space of Disse and additional interactions with immune cells likely enhance deposition of extracellular matrix components and secretion of inhibitors of matrix metalloproteinases cannot be studied in in-vitro systems and therefore represent a significant limitation, particularly when biological processes associated with progressive liver disease are investigated.

\section{Conclusions}

We report genomic responses of primary human hepatocyte and hepatoma cell cultures to the condition of steatosis. We observed regulation of genes mechanistically linked to lipid droplet formation and NAFLD associated changes in mitochondrial lipid and glucose metabolism. 
Our research identified LD associated putative drug targets and among the most promising candidates are the CIDE and perilipin family of proteins. Likewise, proteins of the SNARE complex, i.e. SNAP23 and the vesicle-associated membrane protein 3 as well as the endoplasmic reticulum lipid raft-associated protein 1 are bona fide targets. Collectively, lipid droplet associated proteins can be explored for therapeutic intervention strategies, and the cell culture models proofs to be valuable for an identification of putative targets and an evaluation of drug candidates for the treatment of NAFLD.

\section{Additional files}

\section{Additional file 1: Table S1. Patient characteristics of hepatocyte donors.} (PDF $170 \mathrm{~kb}$ )

Additional file 2: Figure S1. Quantification of intracellular lipid content over time. (A) The plotted graph depicts three treatment conditions, i.e. HepG2 cells without any treatment (K-) or after treatment with the DMSO vehicle control $\left(K_{+}\right)$or after treatment with a 1:1 mixture of the fatty acids OA/PA for $1 \mathrm{~h}, 2 \mathrm{~h}, 4 \mathrm{~h}, 6 \mathrm{~h}, 24 \mathrm{~h}, 48 \mathrm{~h}$ and $72 \mathrm{~h}$. After $6 \mathrm{~h}$ of treatment, the lipid content increased by $25 \mu \mathrm{g} / \mathrm{ml}(\mathrm{K}+, n=28 ; \mathrm{PA} / \mathrm{OA}$, $n=23)$; after $24 \mathrm{~h}$ by $24 \mu \mathrm{g} / \mathrm{ml}(\mathrm{K}+, n=15 ; \mathrm{PA} / \mathrm{OA}, n=18)$, after $48 \mathrm{~h}$ by $70 \mu \mathrm{g} / \mathrm{ml}(\mathrm{K}+, n=14 ; \mathrm{PA} / \mathrm{OA}, n=17)$ and after $72 \mathrm{~h}$ by $71 \mu \mathrm{g} / \mathrm{ml}(\mathrm{K}+$, $n=7 ; \mathrm{PA} / \mathrm{OA}, \mathrm{n}=14)$ when compared to the DMSO vehicle control $(\mathrm{K}+)$, respectively. (T-Test $\mathrm{K}+\leftrightarrow \mathrm{PA} / \mathrm{OA}: 1 \mathrm{~h}, p \leq 0,00 ; 2 \mathrm{~h}, p \leq 1,87 \times 10^{-6} ; 4 \mathrm{~h}$, $p \leq 3,5 \times 10^{-7} ; 6 \mathrm{~h}, p \leq 6,3 \times 10^{-10} ; 24 \mathrm{~h}, p \leq 2,4 \times 10^{-7} ; 48 \mathrm{~h}, \mathrm{p} \leq 2,6 \times 10^{-11}$ $\left.72 \mathrm{~h}, p \leq 1,6 \times 10^{-11}\right)$. * corresponds to a $p$-value of $p \leq 0,001$. (B) The histogram visualizes the increase in intracellular lipid content over time as compared to the DMSO vehicle control. (PDF $356 \mathrm{~kb}$ )

Additional file 3: Figure S2. Live cell imaging of LD fusion. Live cell images of $\mathrm{HuH7}$ cells treated with $0.5 \mathrm{mM}$ of 1:1 mixture of oleic acid and palmitic acid for $24 \mathrm{~h}$. The smaller LDs (orange arrows) appear to fuse with larger LDs (red arrows). Panel B represents the initiation of fusion events where two LDs $\left(v=0.86 \mu \mathrm{m}^{3} ; v=2.9 \mu \mathrm{m}^{3}\right)$ and LDs $\left(v=1.49 \mu \mathrm{m}^{3} ; v=6.88 \mu^{3}\right)$ fuse to form a larger LD $\left(v=3.2 \mu \mathrm{m}^{3}\right)$ and $\left(v=8.78 \mu \mathrm{m}^{3}\right)$, respectively (panel D). The figures were captured at $40 \mathrm{x}$ using time-lapse z-stack by phase contrast microscopy (Nikon TiE) and processed using the NIS elements software version 4.13. The lipid droplets were stained with the blue fluorescent MDP dye (images are not shown). The size bar refers to $10 \mu \mathrm{m}$. (PDF $192 \mathrm{~kb}$ )

Additional file 4: Figure S3. Live cell imaging of LD growth induced by fusogen. (A) Depicted are live cell images of HepG2 cells either (A1) without any treatment (K-) or (A2) treatment with the DMSO vehicle control. (A3) treatment with a $0.5 \mathrm{mM}$ mixture of oleic acid and palmitic acid for $24 \mathrm{~h}$; (A4) FA and $200 \mu \mathrm{M}$ propranolol treatment for $3 \mathrm{~h}$. The live cell images were captured at 40x using time-lapse z-stack by phase contrast fluorescence microscopy (Nikon TiE) and further analyzed using the NIS elements software version 4.13. (B) Depicted are live cell images of LD in HepG2 cells. Upon propranolol treatment the smaller LDs (orange arrows) appear to fuse with larger LDs (red arrows). The insets represent actively grown LDs. (B1) Clockwise: $r=9.14 \mu \mathrm{m}, 12.79,11.34$, 7.9, 8.94, 9.04, 10.26, 11.38, 11.09, 10.28, 8.93. (B2) $r=12.41,11.36,12.46$, 10.88; (B3) $r=16.33,14.21,11.11,17.36$; (B4) $r=19.25,15.88,19.54)$. (B5) The fusion events did not result in spherical lipid droplets; hence the volume could not be determined. The figures were captured at 40x using time-lapse phase contrast microscopy (Nikon TiE) and further processed using the NIS elements software version 4.13. The lipid droplets were stained with the blue fluorescent MDP dye (images are not shown). The size bar refers to $10 \mu \mathrm{m}$. (PDF $417 \mathrm{~kb}$ )

Additional file 5: Table S2. List of mechanistically linked steatotic genes. (PDF $185 \mathrm{~kb}$ )

Additional file 6: Table S3. Compilation of NAFLD responsive genes in in-vitro/in-vivo comparisons. (PDF $96 \mathrm{~kb}$ )

\section{Abbreviations}

ACSL1/ACSL4: Acyl-CoA synthetase long-chain family member; AMPK: 5' adenosine monophosphate-activated protein kinase; ANGPTL4: Angiopoietinlike 4; ANXA1/ANXA2: Annexin A; ARG1: Arginase 1; ATP: Adenosine triphosphate; CD8: Cluster of differentiation 8; CIDEC: Cell Death-Inducing DFFA-Like Effector C; CPT1a/CPT1A: Carnitine palmitoyltransferase; CXCL8: Chemokine (C-X-C Motif) Ligand 8; CYP24A1: Cytochrome P450, family 24, subfamily A, polypeptide 1; CYP26A1: Cytochrome P450, family 26, subfamily A, polypeptide 1; CYP26B1: Cytochrome P450, family 26, subfamily B, polypeptide 1; DAPI: 4',6-Diamidin-2-phenylindol; DEG: Differentially expressed gene; DMEM: Dulbecco's modified media; DMSO: Dimethyl sulfoxide; DTT: Dithiotriol; DYNC1L1: Light intermediate chains;

ER: Endoplasmic reticulum; FA: Fatty acid; FATP2: Fatty acid transport protein 2; FDR: False discovery rate; FGF21: Fibroblast growth factor 21; FOXA1: Forkhead box A1; GLUT2: Glucose transporter 2; GRB2: Growth factor receptor-bound protein 2; GST: Glutathione-S-transferase; GTP: Hydrolyze guanosine triphosphate; HCC: Hepatocellular carcinoma; HIF1a: Hypoxia inducible factor 1, alpha; HSDL2: Hydroxysteroid dehydrogenase like 2; LAMB1: Laminin, Beta 1; LCFA: Long-chain fatty acid; LDLR: Low density lipoprotein receptor; MAP3K8: Mitogen-activated protein kinase 8; MAP4K4: Mitogen-activated protein kinase 4; MDP: Monodansyl pentane; MTTP: Microsomal triglyceride transfer protein; MUFA: Monounsaturated fatty acids; NR1H4: Nuclear receptor subfamily 1, group $\mathrm{H}$, member 4; OA: Oleic acid; ORO: Oil red O; PA: Palmitic acid; PBS: Phosphate-buffered saline; PDK4: Pyruvate dehydrogenase kinase, isozyme 4; PKLR: Pyruvate kinase, liver and RBC (red blood cells); PLIN2: Perilipin 2; PPAR: Peroxisome proliferatoractivated receptor; $\mathrm{RHOB}$ : Ras homolog family member B; RMA: Robust multi-array average; ROS: Reactive oxygen species; SCD: Stearoyl-coA desaturase; SIRT1: Sirtuin1; SLC2A2: Solute carrier family 2; SNAP23: Synaptosomal-Associated Protein, 23 kDa; SNARE: Soluble NSF attachment protein receptor; SOCS2/SOCS3: Suppressor of cytokine signaling; SREBP1: Sterol regulatory element-binding protein 1; TG: Triglycerides; TNFa: Tumor necrosis factor alpha; VLDL: Very low density lipoprotein

\section{Acknowledgements}

The technical assistance of Kerstin Wiesner in performing RNA isolation and microarray experiments is gratefully acknowledged. We also gratefully acknowledge the technical support of Mr. Dario Rizzotto in the Western blotting of PLIN2, respectively.

\section{Funding}

The financial support from The Virtual Liver Network (grant 031 6154) of the German Federal Ministry of Education and Research (BMBF) to JB is gratefully acknowledged. Christin Wallstab was supported by DFG-Graduiertenkolleg 1772- Research Training Group "Computational Systems Biology". The funders had no role in study design, data collection and analysis, decision to publish, or preparation of the manuscript.

\section{Availability of data and materials}

All data generated or analyzed during this study are included in this published article and its supplementary information files. The microarray data is available through the GEO Omnibus public repository (accession number: GSE122660).

\section{Authors' contributions}

SBE performed the microarray experiments, NS and AT performed cell culture, immunofluorescence microscopy and lipid droplet image analysis, CW performed cell culture and lipid droplet image analysis, SBE, NS and JB performed data analysis, all authors discussed the findings. JB wrote the manuscript, all authors have read and approved the manuscript.

\section{Ethics approval and consent to participate}

Liver specimens from $N=6$ patients undergoing elective surgery for colorectal liver metastasis were used to isolate hepatocytes for their culture. All tissue donors gave informed consent for experimental use of clinical data and liver specimen prior to surgery. Ethical approval for the protocol and use liver samples was obtained from the Ethics Committee of the Hannover Medical School (Tr/L, 2499 and $\mathrm{Tr} / \mathrm{L}, 466$ 31,309). 


\section{Competing interests}

The authors have declared that no competing interests exist.

\section{Publisher's Note}

Springer Nature remains neutral with regard to jurisdictional claims in published maps and institutional affiliations.

\section{Author details}

'Centre for Pharmacology and Toxicology, Hannover Medical School, Carl-Neuberg-Str. 1, 30625 Hannover, Germany. ${ }^{2}$ Institute of Biochemistry, Charité - University Medicine Berlin, Charitéplatz 1, 10117 Berlin, Germany.

\section{Received: 11 September 2018 Accepted: 23 November 2018}

\section{Published online: 14 December 2018}

\section{References}

1. Brunt EM, Tiniakos DG. Histopathology of nonalcoholic fatty liver disease. World J Gastroenterol. 2010;16:5286-96.

2. Review Team, LaBrecque DR, Abbas Z, Anania F, Ferenci P, Khan AG, et al. World gastroenterology organisation global guidelines: nonalcoholic fatty liver disease and nonalcoholic steatohepatitis. J Clin Gastroenterol. 2014;48:467-73.

3. Abenavoli L, Milic N, Di Renzo L, Preveden T, Medic-Stojanoska M, De Lorenzo A. Metabolic aspects of adult patients with nonalcoholic fatty liver disease. World J Gastroenterol. 2016;22:7006-16.

4. Sahini N, Borlak J. Recent insights into the molecular pathophysiology of lipid droplet formation in hepatocytes. Prog Lipid Res. 2014:54:86-112.

5. Anderson N, Borlak J. Molecular mechanisms and therapeutic targets in steatosis and steatohepatitis. Pharmacol Rev. 2008:60:311-57.

6. Grundy SM, Brewer HB Jr, Cleeman Jl, Smith SC Jr, Lenfant C, American Heart Association, et al. Definition of metabolic syndrome: Report of the National Heart, Lung, and Blood Institute/American Heart Association conference on scientific issues related to definition. Circulation. 2004;109:433-8.

7. Oliveira CP, Stefano JT. Genetic polymorphisms and oxidative stress in nonalcoholic steatohepatitis (NASH). Clin Res Hepatol Gastroenterol. 2015; 39(Suppl 1):S35-40. Review.

8. Zhang XQ, Xu CF, Yu CH, Chen WX, Li YM. Role of endoplasmic reticulum stress in the pathogenesis of nonalcoholic fatty liver disease. World J Gastroenterol. 2014;20:1768-76.

9. Hassan K, Bhalla V, El Regal ME, A-Kader HH. Nonalcoholic fatty liver disease: a comprehensive review of a growing epidemic. World I Gastroenterol. 2014:20:12082-101.

10. Pessayre D, Mansouri A, Haouzi D, Fromenty B. Hepatotoxicity due to mitochondrial dysfunction. Cell Biol Toxicol. 1999;15:367-73.

11. Imrie D, Sadler KC. Stress management: how the unfolded protein response impacts fatty liver disease. J Hepatol. 2012;57:1147-51.

12. Pagliassotti MJ. Endoplasmic reticulum stress in nonalcoholic fatty liver disease. Annu Rev Nutr. 2012;32:17-33.

13. Thacker SA, Robinson P, Abel A, Tweardy DJ. Modulation of the unfolded protein response during hepatocyte and cardiomyocyte apoptosis in trauma/hemorrhagic shock. Sci Rep. 2013;3:1187.

14. Zhou H, Liu R. ER stress and hepatic lipid metabolism. Front Genet. 2014;5:112.

15. Guo Y, Cordes KR, Farese RV Jr, Walther TC. Lipid droplets at a glance. J Cell Sci. 2009;122:749-52.

16. Walther TC, Farese RV Jr. Lipid droplets and cellular lipid metabolism. Annu Rev Biochem. 2012;81:687-714.

17. D'Aquila T, Sirohi D, Grabowski JM, Hedrick VE, Paul LN, Greenberg AS, et al. Characterization of the proteome of cytoplasmic lipid droplets in mouse enterocytes after a dietary fat challenge. PLoS One. 2015;10:e0126823.

18. Chen E, Tsai TH, Li L, Saha P, Chan L, Chang BH. PLIN2 is a key regulator of the unfolded protein response and endoplasmic reticulum stress resolution in pancreatic beta cells. Sci Rep. 2017;7:40855.

19. Manco M, Marcellini M, Giannone G, Nobili V. Correlation of serum TNFalpha levels and histologic liver injury scores in pediatric nonalcoholic fatty liver disease. Am J Clin Pathol. 2007;127:954-60.

20. Borlak J, Hock A, Hansen T, Richter E. DNA adducts in cultures of polychlorinated biphenyl-treated human hepatocytes. Toxicol Appl Pharmacol. 2003;188:81-91.

21. Borlak J, Chougule A, Singh PK. How useful are clinical liver function tests in in vitro human hepatotoxicity assays? Toxicol in Vitro. 2014;28:784-95.

22. Borlak J, Singh PK, Rittelmeyer I. Regulation of liver enriched transcription factors in rat hepatocytes cultures on collagen and EHS sarcoma matrices. PLoS One. 2015;10:e0124867.
23. Black PN, Ahowesso C, Montefusco D, Saini N, DiRusso CC. Fatty acid transport proteins: targeting FATP2 as a gatekeeper involved in the transport of exogenous fatty acids. Medchemcomm. 2016;7:612-22.

24. Del Vescovo V, Meier T, Inga A, Denti MA, Borlak J. A cross-platform comparison of affymetrix and Agilent microarrays reveals discordant miRNA expression in lung tumors of c-Raf transgenic mice. PLoS One. 2013;8:e78870

25. Anderson N, Meier T, Borlak J. Toxicogenomics applied to cultures of human hepatocytes enabled an identification of novel petasites hybridus extracts for the treatment of migraine with improved hepatobiliary safety. Toxicol Sci. 2009;112:507-20

26. Wilfling F, Wang H, Haas JT, Krahmer N, Gould TJ, Uchida A, et al. Triacylglycerol synthesis enzymes mediate lipid droplet growth by relocalizing from the ER to lipid droplets. Dev Cell. 2013;24:384-99.

27. Xu L, Zhou L, Li P. CIDE proteins and lipid metabolism. Arterioscler Thromb Vasc Biol. 2012:32:1094-8.

28. Murphy S, Martin S, Parton RG. Quantitative analysis of lipid droplet fusion: inefficient steady state fusion but rapid stimulation by chemical fusogens. PLoS One. 2010;5:e15030.

29. McKee C, Soeda J, Asilmaz E, Sigalla B, Morgan M, Sinelli N, et al. Propranolol, a beta-adrenoceptor antagonist, worsens liver injury in a model of non-alcoholic steatohepatitis. Biochem Biophys Res Commun. 2013:437:597-602

30. Ahowesso C, Black PN, Saini N, Montefusco D, Chekal J, Malosh C, et al. Chemical inhibition of fatty acid absorption and cellular uptake limits lipotoxic cell death. Biochem Pharmacol. 2015;98:167-81.

31. Saini N, Black PN, Montefusco D, DiRusso CC. Fatty acid transport protein-2 inhibitor Grassofermata/CB5 protects cells against lipid accumulation and toxicity. Biochem Biophys Res Commun. 2015;465:534-41.

32. Sandoval A, Chokshi A, Jesch ED, Black PN, Dirusso CC. Identification and characterization of small compound inhibitors of human FATP2. Biochem Pharmacol. 2010;79:990-9.

33. Girard E, Paul JL, Fournier N, Beaune P, Johannes L, Lamaze C, et al. The dynamin chemical inhibitor dynasore impairs cholesterol trafficking and sterol-sensitive genes transcription in human HeLa cells and macrophages. PLoS One. 2011;6:e29042.

34. Kirchhausen T, Macia E, Pelish HE. Use of dynasore, the small molecule inhibitor of dynamin, in the regulation of endocytosis. Methods Enzymol. 2008;438:77-93.

35. Newton AJ, Kirchhausen T, Murthy VN. Inhibition of dynamin completely blocks compensatory synaptic vesicle endocytosis. Proc Natl Acad Sci U S A. 2006;103:17955-60.

36. Pohl J, Ring A, Stremmel W. Uptake of long-chain fatty acids in HepG2 cells involves caveolae: analysis of a novel pathway. J Lipid Res. 2002;43:1390-9.

37. Gronemeyer T, Wiese S, Ofman R, Bunse C, Pawlas M, Hayen H, et al. The proteome of human liver peroxisomes: identification of five new peroxisomal constituents by a label-free quantitative proteomics survey. PLoS One. 2013;8:e57395.

38. Paton CM, Ntambi JM. Biochemical and physiological function of stearoylCoA desaturase. Am J Physiol Endocrinol Metab. 2009;297:E28-37.

39. Moya M, Benet M, Guzman C, Tolosa L, Garcia-Monzon C, Pareja E, et al. Foxa1 reduces lipid accumulation in human hepatocytes and is downregulated in nonalcoholic fatty liver. PLoS One. 2012;7:e30014.

40. Liu J, Xu Y, Hu Y, Wang G. The role of fibroblast growth factor 21 in the pathogenesis of non-alcoholic fatty liver disease and implications for therapy. Metabolism. 2015;64:380-90.

41. Eliades M, Spyrou E. Vitamin D: a new player in non-alcoholic fatty liver disease? World J Gastroenterol. 2015;21:1718-27.

42. Inoue T, Kohro T, Tanaka T, Kanki Y, Li G, Poh HM, et al. Cross-enhancement of ANGPTL4 transcription by HIF1 alpha and PPAR beta/delta is the result of the conformational proximity of two response elements. Genome Biol. 2014;15:R63,2014-15-4-r63.

43. Koliwad SK, Kuo T, Shipp LE, Gray NE, Backhed F, So AY, et al. Angiopoietinlike 4 (ANGPTL4, fasting-induced adipose factor) is a direct glucocorticoid receptor target and participates in glucocorticoid-regulated triglyceride metabolism. J Biol Chem. 2009:284:25593-601.

44. Catoire M, Alex S, Paraskevopulos N, Mattijssen F, Evers-van Gogh I, Schaart $\mathrm{G}$, et al. Fatty acid-inducible ANGPTL4 governs lipid metabolic response to exercise. Proc Natl Acad Sci U S A. 2014;111:E1043-52.

45. Lichtenstein L, Berbee JF, van Dijk SJ, van Dijk KW, Bensadoun A, Kema IP, et al. Angpt|4 upregulates cholesterol synthesis in liver via inhibition of LPLand HL-dependent hepatic cholesterol uptake. Arterioscler Thromb Vasc Biol. 2007;27:2420-7. 
46. Coskun T, Bina HA, Schneider MA, Dunbar JD, Hu CC, Chen Y, et al. Fibroblast growth factor 21 corrects obesity in mice. Endocrinology. 2008;149:6018-27.

47. Lee DV, Li D, Yan Q, Zhu Y, Goodwin B, Calle R, et al. Fibroblast growth factor 21 improves insulin sensitivity and synergizes with insulin in human adipose stem cell-derived (hASC) adipocytes. PLoS One. 2014;9:e111767.

48. Zadjali F, Santana-Farre R, Vesterlund M, Carow B, Mirecki-Garrido M, Hernandez-Hernandez I, et al. SOCS2 deletion protects against hepatic steatosis but worsens insulin resistance in high-fat-diet-fed mice. FASEB J. 2012;26:3282-91.

49. Sachithanandan N, Fam BC, Fynch S, Dzamko N, Watt MJ, Wormald S, et al. Liver-specific suppressor of cytokine signaling-3 deletion in mice enhances hepatic insulin sensitivity and lipogenesis resulting in fatty liver and obesity. Hepatology. 2010;52:1632-42.

50. Marra F, Tacke F. Roles for chemokines in liver disease. Gastroenterology. 2014;147:577-594.e1.

51. Xu L, Kitade H, Ni Y, Ota T. Roles of chemokines and chemokine receptors in obesity-associated insulin resistance and nonalcoholic fatty liver disease. Biomol Ther. 2015;5:1563-79.

52. Chowdhury FZ, Estrada LD, Murray S, Forman J, Farrar JD. Pharmacological inhibition of TPL2/MAP3K8 blocks human cytotoxic T lymphocyte effector functions. PLoS One. 2014;9:e92187.

53. Danai LV, Flach RJ, Virbasius JV, Menendez LG, Jung DY, Kim JH, et al. Inducible deletion of protein kinase Map4k4 in obese mice improves insulin sensitivity in liver and adipose tissues. Mol Cell Biol. 2015;35:2356-65.

54. Sahini N, Borlak J. Genomics of human fatty liver disease reveal mechanistically linked lipid droplet-associated gene regulations in blunt steatosis and nonalcoholic steatohepatitis. Transl Res. 2016;177:41-69.

55. D'souza AM, Beaudry JL, Szigiato AA, Trumble SJ, Snook LA, Bonen A, et al. Consumption of a high-fat diet rapidly exacerbates the development of fatty liver disease that occurs with chronically elevated glucocorticoids. Am J Physiol Gastrointest Liver Physiol. 2012;302:G850-63.

56. Anderson N, Borlak J. Drug-induced phospholipidosis. FEBS Lett. 2006;580: 5533-40.

57. Loudig O, Babichuk C, White J, Abu-Abed S, Mueller C, Petkovich M. Cytochrome P450RAI(CYP26) promoter: a distinct composite retinoic acid response element underlies the complex regulation of retinoic acid metabolism. Mol Endocrinol. 2000;14:1483-97.

58. White JA, Ramshaw H, Taimi M, Stangle W, Zhang A, Everingham S, et al. Identification of the human cytochrome P450, P450RAI-2, which is predominantly expressed in the adult cerebellum and is responsible for alltrans-retinoic acid metabolism. Proc Natl Acad Sci U S A. 2000;97:6403-8.

59. Jaeschke H, Gores GJ, Cederbaum Al, Hinson JA, Pessayre D, Lemasters JJ. Mechanisms of hepatotoxicity. Toxicol Sci. 2002;65:166-76.

60. Bechmann LP, Hannivoort RA, Gerken G, Hotamisligil GS, Trauner M, Canbay A. The interaction of hepatic lipid and glucose metabolism in liver diseases. J Hepatol. 2012;56:952-64.

61. Kim S, Jung J, Kim H, Heo RW, Yi CO, Lee JE, et al. Exendin-4 improves nonalcoholic fatty liver disease by regulating glucose transporter 4 expression in Ob/Ob mice. Korean J Physiol Pharmacol. 2014;18:333-9.

62. Im SS, Kang SY, Kim SY, Kim HI, Kim JW, Kim KS, et al. Glucose-stimulated upregulation of GLUT2 gene is mediated by sterol response elementbinding protein-1c in the hepatocytes. Diabetes. 2005;54:1684-91.

63. Zhu S, Ma L, Wu Y, Ye X, Zhang T, Zhang Q, et al. FGF21 treatment ameliorates alcoholic fatty liver through activation of AMPK-SIRT1 pathway. Acta Biochim Biophys Sin Shanghai. 2014;46:1041-8.

64. Senthivinayagam S, Mclntosh AL, Moon KC, Atshaves BP. Plin2 inhibits cellular glucose uptake through interactions with SNAP23, a SNARE complex protein. PLoS One. 2013;8:e73696.

65. Bostrom P, Andersson L, Rutberg M, Perman J, Lidberg U, Johansson BR, et al. SNARE proteins mediate fusion between cytosolic lipid droplets and are implicated in insulin sensitivity. Nat Cell Biol. 2007:9:1286-93.

66. Watkins PA, Ellis JM. Peroxisomal acyl-CoA synthetases. Biochim Biophys Acta. 1822:2012:1411-20

67. Hinder LM, Figueroa-Romero C, Pacut C, Hong Y, Vivekanandan-Giri A, Pennathur $\mathrm{S}$, et al. Long-chain acyl coenzyme a synthetase 1 overexpression in primary cultured Schwann cells prevents long chain fatty acid-induced oxidative stress and mitochondrial dysfunction. Antioxid Redox Signal. 2014;21:588-600.

68. Ellis JM, Frahm JL, Li LO, Coleman RA. Acyl-coenzyme a synthetases in metabolic control. Curr Opin Lipidol. 2010;21:212-7.
69. Schrem H, Klempnauer J, Borlak J. Liver-enriched transcription factors in liver function and development. Part I: the hepatocyte nuclear factor network and liver-specific gene expression. Pharmacol Rev. 2002:54:129-58.

70. Braunersreuther V, Viviani GL, Mach F, Montecucco F. Role of cytokines and chemokines in non-alcoholic fatty liver disease. World J Gastroenterol. 2012;18:727-35.

71. Anderson CM, Stahl A. SLC27 fatty acid transport proteins. Mol Asp Med. 2013:34:516-28.

72. Stahl A, Gimeno RE, Tartaglia LA, Lodish HF. Fatty acid transport proteins: a current view of a growing family. Trends Endocrinol Metab. 2001;12:266-73

73. Marina-Garcia N, Franchi L, Kim YG, Hu Y, Smith DE, Boons GJ, et al. Clathrinand dynamin-dependent endocytic pathway regulates muramyl dipeptide internalization and NOD2 activation. J Immunol. 2009;182:4321-7.

74. Bedu E, Wahli W, Desvergne B. Peroxisome proliferator-activated receptor beta/delta as a therapeutic target for metabolic diseases. Expert Opin Ther Targets. 2005;9:861-73.

75. Tso SC, Qi X, Gui WJ, Wu CY, Chuang JL, Wernstedt-Asterholm I, et al. Structure-guided development of specific pyruvate dehydrogenase kinase inhibitors targeting the ATP-binding pocket. J Biol Chem. 2014:289:4432-43.

76. Padmanabha Das KM, Wechselberger L, Liziczai M, De la Rosa RM, Grabner GF, Heier C, et al. Hypoxia-inducible lipid droplet-associated protein inhibits adipose triglyceride lipase. J Lipid Res. 2018;59:531-41.

77. Hack K, Reilly L, Palmer C, Read KD, Norval S, Kime R, et al. Skintargeted inhibition of PPAR beta/delta by selective antagonists to treat PPAR beta/delta-mediated psoriasis-like skin disease in vivo. PLoS One. 2012;7:e37097.

78. Xiao W, Ren M, Zhang C, Li S, An W. Amelioration of nonalcoholic fatty liver disease by hepatic stimulator substance via preservation of carnitine palmitoyl transferase-1 activity. Am J Physiol Cell Physiol. 2015;309:C215-27.

79. Monsenego J, Mansouri A, Akkaoui M, Lenoir V, Esnous C, Fauveau V, et al. Enhancing liver mitochondrial fatty acid oxidation capacity in obese mice improves insulin sensitivity independently of hepatic steatosis. J Hepatol. 2012;56:632-9.

80. Ward C, Martinez-Lopez N, Otten EG, Carroll B, Maetzel D, Singh R, et al. Autophagy, lipophagy and lysosomal lipid storage disorders. Biochim Biophys Acta. 1861;2016:269-84.

81. Liu L, Zheng LD, Zou P, Brooke J, Smith C, Long YC, et al. FoxO1 antagonist suppresses autophagy and lipid droplet growth in adipocytes. Cell Cycle. 2016;15:2033-41.

82. Pandey A, Kumar GS, Kadakol A, Malek V, Gaikwad AB. FoxO1 inhibitors: the future medicine for metabolic disorders? Curr Diabetes Rev. 2016;12:223-30

83. Boettcher E, Csako G, Pucino F, Wesley R, Loomba R. Meta-analysis: pioglitazone improves liver histology and fibrosis in patients with nonalcoholic steatohepatitis. Aliment Pharmacol Ther. 2012;35:66-75.

84. Goh VJ, Silver DL. The lipid droplet as a potential therapeutic target in NAFLD. Semin Liver Dis. 2013;33:312-20.

85. Noureldein $\mathrm{MH}$. In silico discovery of a perilipin 1 inhibitor to be used as a new treatment for obesity. Eur Rev Med Pharmacol Sci. 2014;18:457-60.

86. Conte M, Franceschi C, Sandri M, Salvioli S. Perilipin 2 and age-related metabolic diseases: a new perspective. Trends Endocrinol Metab. 2016:27:893-903.

87. Riento K, Frick M, Schafer I, Nichols BJ. Endocytosis of flotillin-1 and flotillin-2 is regulated by Fyn kinase. J Cell Sci. 2009:122:912-8.

88. Kharitonenkov A, DiMarchi R. FGF21 revolutions: recent advances illuminating FGF21 biology and medicinal properties. Trends Endocrinol Metab. 2015;26:608-17.

89. Zhang J, Li Y. Fibroblast Growth Factor 21 Analogs for Treating Metabolic Disorders. Front Endocrinol (Lausanne). 2015;6:168.

90. Preta G, Cronin JG, Sheldon IM. Dynasore - not just a dynamin inhibitor. Cell Commun Signal. 2015;13(24):015-0102-1.

91. Masuda T, Ogura MN, Moriya T, Takahira N, Matsumoto T, Kutsuna T, et al. Beneficial effects of $\mathrm{L}$ - and $\mathrm{N}$-type calcium channel blocker on glucose and lipid metabolism and renal function in patients with hypertension and type II diabetes mellitus. Cardiovasc Ther. 2011;29:46-53. 